\title{
A New Class of Difference Methods with Intrinsic Parallelism for Burgers-Fisher Equation
}

\author{
Yueyue Pan $\mathbb{D}^{1},{ }^{1}$ Lifei Wu $\mathbb{D},^{2}$ and Xiaozhong Yang $\mathbb{D}^{2}$ \\ ${ }^{1}$ School of Control and Computer Engineering, North China Electric Power University, Beijing 102206, China \\ ${ }^{2}$ School of Mathematics and Physics, North China Electric Power University, Beijing 102206, China \\ Correspondence should be addressed to Xiaozhong Yang; yxiaozh@ncepu.edu.cn
}

Received 11 February 2020; Revised 26 May 2020; Accepted 30 June 2020; Published 14 August 2020

Academic Editor: Libor Pekař

Copyright (c) 2020 Yueyue Pan et al. This is an open access article distributed under the Creative Commons Attribution License, which permits unrestricted use, distribution, and reproduction in any medium, provided the original work is properly cited.

This paper proposes a new class of difference methods with intrinsic parallelism for solving the Burgers-Fisher equation. A new class of parallel difference schemes of pure alternating segment explicit-implicit (PASE-I) and pure alternating segment implicitexplicit (PASI-E) are constructed by taking simple classical explicit and implicit schemes, combined with the alternating segment technique. The existence, uniqueness, linear absolute stability, and convergence for the solutions of PASE-I and PASI-E schemes are well illustrated. Both theoretical analysis and numerical experiments show that PASE-I and PASI-E schemes are linearly absolute stable, with 2-order time accuracy and 2-order spatial accuracy. Compared with the implicit scheme and the Crank-Nicolson (C-N) scheme, the computational efficiency of the PASE-I (PASI-E) scheme is greatly improved. The PASE-I and PASI-E schemes have obvious parallel computing properties, which show that the difference methods with intrinsic parallelism in this paper are feasible to solve the Burgers-Fisher equation.

\section{Introduction}

The Burgers-Fisher equation is a typical model for describing diffusion propagation and convection conduction. It is an important partial differential equation in mathematical physics and widely applied not only in the study of gas dynamics and heat conduction, but also in explaining many physical phenomena such as elasticity [1-3]. In recent years, the numerical method for solving the Burgers-Fisher equation has been extensively concerned by researchers [4-6]. They study this equation both for conceptual understanding of physical flows and testing various numerical methods. So, the fast method of solving it has basic scientific significance and application value.

Up to now, researchers have given many numerical methods for solving the Burgers-Fisher equation. Ismail et al. [7] obtained the approximate solution of the Burgers-Fisher equation by using the Adomian decomposition method. Although the approximate solution had high accuracy, the method neither allows to choose different basis functions, nor could it adjust the convergence region and speed of the asymptotic solution. Babolian and Saeidian [8] applied the homotopy analysis method to give the analytical approximate solution of the Burgers-Fisher equation. This method is effective for solving such partial differential equations. However, auxiliary operators, auxiliary parameters, and auxiliary functions have been introduced in a wide range, and there is no strict mathematical theory to guide their selection to ensure the convergence of numerical solution series. Chandraker et al. [9] proposed a semi-implicit difference scheme and a modified C-N difference method to solve the Burgers-Fisher equation. For this method, numerical solution errors are much less, but the stability of the semi-implicit method is weak, and it is conditionally stable. Yadav and Jiwari [10] presented finite element analysis and approximation of the Burgers-Fisher equation, which proves that the scheme is convergent. However, the calculated amount of the method is very large, and its parallel calculation is not as intuitive as the finite difference method. And, the computational efficiencies, especially the computation time, of these methods in the above documents are low. For the high-dimensional Burgers-Fisher equation, 
even with high-performance computers, it is difficult to simulate a large computational domain.

With the rapid development of multicore and cluster technology, the parallel algorithm has become one of the mainstream technologies to improve the computing efficiency. It is well known that classical explicit difference schemes have ideal parallelism and are suitable for parallel computation, but they are conditionally stable. Especially in multidimensional problems, the time step of computing is severely restricted. The classical implicit difference scheme and the $\mathrm{C}-\mathrm{N}$ difference scheme are absolutely stable, but they are not suitable for direct and effective application on parallel computers. For parabolic equations, Evans and Abdullah [11] proposed the idea of group explicit and designed an alternating group explicit (AGE) difference scheme, which not only ensures the stability of numerical calculations, but also has a good parallel property. Inspired by the construction of the AGE method, Zhang et al. $[12,13]$ proposed the idea of using the Saul'yev asymmetric scheme to construct a piecewise implicit scheme and established a class of alternating segment explicit-implicit (ASE-I) parallel difference methods and alternating segment C-N (ASC-N) parallel difference methods, where the stability and parallelism were both obtained. Han et al. [14] constructed pure alternating segment explicit-implicit (PASE-I) and pure alternating segment implicit-explicit (PASI-E) parallel difference schemes for the constant coefficient diffusion equation. The numerical solution had good stability and convergence. Academician Zhou [15] referred the explicitimplicit mixing scheme of the most general parabolic equation as the difference scheme with intrinsic parallelism. $\mathrm{He}$ studied the existence, uniqueness, convergence, and stability of differential decomposition and established the basic theory of the difference method with intrinsic parallelism for parabolic equations. Until now, the difference method with intrinsic parallelism has been extended to solve many evolution equations [16-18]. Qu and Wang [19] constructed an alternating segment explicit-implicit difference scheme for solving the KdV equation. The linear absolute stability of the scheme has also been demonstrated. Yuan et al. [20] proposed a class of parallel difference schemes with 2-order spatial accuracy and unconditional stability for the nonlinear parabolic system. Guo et al. [21] studied the difference method with intrinsic parallelism for the dispersive equation. The general alternating difference schemes with variable time steps are constructed and proved to be unconditionally stable. Namjoo et al. [22] presented the numerical solution of the generalized Burgers-Fisher equation on the basis of the nonstandard finite-difference (NSFD) scheme. The positivity, consistency, and boundedness of the scheme are discussed. The numerical results obtained by the NSFD scheme is compared with the exact solution and some available methods to verify the accuracy and efficiency of the NSFD scheme. For the nonlinear Leland equation, it is very difficult to obtain an analytical solution. Therefore, let the numerical solution of the C-N scheme approximately substitute the exact solution. Yan et al. [23] constructed PASE-I and PASI-E difference schemes, which combine the classical explicit scheme with the classical implicit scheme. The numerical experiments verify that the calculation accuracy of PASE-I and PASI-E schemes is better than that of the existing alternating segment $\mathrm{C}-\mathrm{N}$ scheme and alternating segment explicit-implicit and implicit-explicit schemes. The main advantage of these difference schemes with intrinsic parallelism is that these methods can be directly applied to parallel computer systems with distributed memory and minimize the communication between processors. The algorithm only needs local message passing between adjacent processors, and the communication and computation involved are also local. It is easy to balance the load of computation and communication, so as to obtain good precision and extendibility of parallel computing.

For a long time, a large number of parallel difference schemes constructed are either conditionally stable or unconditionally stable, but the space has only 1-order accuracy [24]. To obtain a parallel difference scheme with higher precision and more relaxed stability conditions, we study the parallel algorithm of the Burgers-Fisher equation from the point of view of the parallelization of traditional difference schemes. We hope to skip the difficulties of the numerical algebra and open up another way of parallelization. In this paper, a new class of difference method with intrinsic parallelism for the Burgers-Fisher equation is proposed by making piecewise processing of alternating whole explicit and implicit difference schemes. A new class of PASE-I and PASI-E parallel difference schemes are constructed by taking simple classical explicit and implicit schemes. Both numerical experiments and theoretical analysis show that PASE-I and PASI-E schemes have obvious parallel computational properties. The schemes show linear absolute stability and convergence with 2-order time accuracy and 2order spatial accuracy. Numerical examples give that the computational efficiencies of PASE-I and PASI-E difference schemes are much higher than those of the implicit difference scheme. And, the parallel difference methods proposed in this paper are efficient and feasible for solving the Burgers-Fisher equation.

\section{The Intrinsic Parallel Difference Schemes of Burgers-Fisher Equation}

2.1. Burgers-Fisher Equation. The general form of the Burgers-Fisher equation is $[1,2]$

$$
\frac{\partial u}{\partial t}+\alpha u^{\delta} \frac{\partial u}{\partial x}=\mu \frac{\partial^{2} u}{\partial x^{2}}+\beta u\left(1-u^{\delta}\right), \quad 0 \leq x \leq L, 0 \leq t \leq T,
$$

where $\alpha>0, \delta>0, \mu>0$, and $\beta<0$ are constants.

The initial condition is

$$
u(x, 0)=f(x), \quad 0 \leq x \leq L .
$$

The boundary conditions are

$$
\begin{gathered}
u(0, t)=g_{1}(t), \\
u(L, t)=g_{2}(t), \\
0<t \leq T .
\end{gathered}
$$


Taking the determined $f(x), g_{1}(t)$, and $g_{2}(t)$, respectively, as

$$
\begin{aligned}
& f(x)=\left[\frac{1}{2}+\frac{1}{2} \tanh \left(\frac{-\alpha \delta x}{2(1+\delta)}\right)\right]^{1 / \delta}, \\
& g_{1}(t)=\left[\frac{1}{2}+\frac{1}{2} \tanh \left(\frac{-\alpha \delta}{2(1+\delta)}\left(-\left(\frac{\alpha}{1+\delta}+\frac{\beta(1+\delta)}{\alpha}\right) t\right)\right)\right]^{1 / \delta}, \\
& g_{2}(t)=\left[\frac{1}{2}+\frac{1}{2} \tanh \left(\frac{-\alpha \delta}{2(1+\delta)}\left(L-\left(\frac{\alpha}{1+\delta}+\frac{\beta(1+\delta)}{\alpha}\right) t\right)\right)\right]^{1 / \delta} .
\end{aligned}
$$

We can get the analytic solution:

$$
u(x, t)=\left[\frac{1}{2}+\frac{1}{2} \tanh \left(\frac{-\alpha \delta}{2(1+\delta)}\left(x-\left(\frac{\alpha}{1+\delta}+\frac{\beta(1+\delta)}{\alpha}\right) t\right)\right)\right]^{1 / \delta}
$$

\subsection{The Construction of PASE-I Parallel Difference Scheme.} The solution region $[0, L] \times[0, T]$ is divided into grids. Respectively, the space and time steps are $\Delta x=(L / M)$ and $\Delta t$, where $M$ is a positive integer. Here, $x_{i}=i \Delta x(i=$ $0,1,2 \ldots, M)$ and $t_{n}=n \Delta t(n=0,1,2, \ldots) . u_{i}^{n}$ is the numerical solution, and $U\left(x_{i}, t_{n}\right)$ is the analytic solution. To construct the PASE-I parallel difference scheme, the classical explicit difference scheme, the classical implicit difference scheme, and the $\mathrm{C}-\mathrm{N}$ difference scheme of equation (1) will be given first.

At first, the classical explicit difference scheme is

$$
u_{i}^{n+1}=\left(r_{1}+r_{2}\right) u_{i-1}^{n}+\left(1-2 r_{1}+r_{3}\right) u_{i}^{n}+\left(r_{1}-r_{2}\right) u_{i+1}^{n} \text {. }
$$

Secondly, the classical implicit difference scheme is

$$
\left(-r_{1}-r_{2}\right) u_{i-1}^{n+1}+\left(1+2 r_{1}-r_{3}\right) u_{i}^{n+1}+\left(-r_{1}+r_{2}\right) u_{i+1}^{n+1}=u_{i}^{n} \text {. }
$$

Thirdly, the C-N difference scheme is

$$
\begin{gathered}
\left(-\frac{r_{1}}{2}-\frac{r_{2}}{2}\right) u_{i-1}^{n+1}+\left(1+r_{1}-\frac{r_{3}}{2}\right) u_{i}^{n+1}+\left(-\frac{r_{1}}{2}+\frac{r_{2}}{2}\right) u_{i+1}^{n+1} \\
=\left(\frac{r_{1}}{2}+\frac{r_{2}}{2}\right) u_{i-1}^{n}+\left(1-r_{1}+\frac{r_{3}}{2}\right) u_{i}^{n}+\left(\frac{r_{1}}{2}-\frac{r_{2}}{2}\right) u_{i+1}^{n},
\end{gathered}
$$

where $\quad r_{1}=\left(\mu \Delta t /(\Delta x)^{2}\right), \quad r_{2}=\left(\alpha a_{i}^{n} \Delta t / 2 \Delta x\right), \quad r_{3}=\beta(1-$ $\left.a_{i}^{n}\right) \Delta t, a_{i}^{n}=\left(1 / 4\left(u_{i-1}^{n}+2 u_{i}^{n}+u_{i+1}^{n}\right)\right)^{\delta}$, and $u^{\delta}=a_{i}^{n}$. The discrete schemes of $u^{\delta}(\partial u / \partial x)$ are $\left(1 / 4\left(u_{i-1}^{n}+2 u_{i}^{n}+\right.\right.$ $\left.\left.u_{i+1}^{n}\right)\right)^{\delta}\left(\left(u_{i+1}^{n}-u_{i-1}^{n}\right) / 2 \Delta x\right)$ in the explicit scheme and $(1 / 4$ $\left.\left(u_{i-1}^{n}+2 u_{i}^{n}+u_{i+1}^{n}\right)\right)^{\delta}\left(\left(u_{i+1}^{n+1}-u_{i-1}^{n+1}\right) / 2 \Delta x\right)$ in the implicit scheme.

The classical explicit scheme has ideal parallelism, but it is conditionally stable. The classical implicit scheme is absolutely stable, but the inverse matrix of the tridiagonal matrix needs to be solved, so it is not convenient for us to get the results directly and quickly. So, combining the classical explicit difference scheme (6) with the classical implicit difference scheme (7), the specific approach is given as follows.

Let $M-1=J l$, where $J$ and $l$ are positive integers, $J$ is odd, and $J \geq 3$ and $l \geq 3$. The points calculated at the same time layer are divided into $J$ segments, which are recorded in the order $S_{1}, S_{2}, \ldots, S_{J}$. Every segment of the odd time layer is arranged from left to right in the order of "classical explicit-classical implicit-classical explicit." On the even time layer, the order of arrangement becomes "classical implicitclassical explicit-classical implicit." The solution of each implicit segment relies on the calculation of the first or last point of the adjacent explicit segment to give its internal boundary value. See Figure 1 for the detail, where the classical explicit scheme is applied in the $\hat{\mathrm{x}}$ place and the classical implicit scheme is applied in the $\square$ place.

For realizing the parallel computing of the PASE-I scheme, for $i_{0} \geq 0$, we consider the calculation of the explicit segment point $\left(i_{0}+i, n+1\right), i=1,2, \ldots, l$. They are calculated with the classical explicit scheme (6), leading to the following explicit segment:

$$
\begin{aligned}
& \left(\begin{array}{c}
u_{i_{0}+1}^{n+1} \\
u_{i_{0}+2}^{n+1} \\
\vdots \\
u_{i_{0}+l-1}^{n+1} \\
u_{i_{0}+l}^{n+1}
\end{array}\right)_{l \times 1}=\left(\begin{array}{ccccc}
1-2 r_{1}+r_{3} & r_{1}-r_{2} & & \\
r_{1}+r_{2} & 1-2 r_{1}+r_{3} & r_{1}-r_{2} & \\
& \ddots & \ddots & \ddots & \\
& & r_{1}+r_{2} & 1-2 r_{1}+r_{3} & r_{1}-r_{2} \\
& & & r_{1}+r_{2} & 1-2 r_{1}+r_{3}
\end{array}\right)_{l \times l} \\
& \left(\begin{array}{c}
u_{i_{0}+1}^{n} \\
u_{i_{0}+2}^{n} \\
\vdots \\
u_{i_{0}+l-1}^{n} \\
u_{i_{0}+l}^{n}
\end{array}\right)_{l \times 1}+\left(\begin{array}{c}
\left(r_{1}+r_{2}\right) u_{i_{0}}^{n} \\
\vdots \\
\left(r_{1}-r_{2}\right) u_{i_{0}+l+1}^{n}
\end{array}\right)_{l \times 1}
\end{aligned}
$$




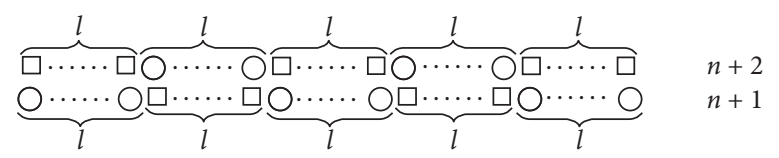

FIgURE 1: Structural schematic diagram of the PASE-I scheme $(J=5)$.

The implicit segment is

$$
\begin{aligned}
& \left(\begin{array}{ccccc}
1+2 r_{1}-r_{3} & -r_{1}+r_{2} & & & \\
-r_{1}-r_{2} & 1+2 r_{1}-r_{3} & -r_{1}+r_{2} & & \\
& \ddots & \ddots & \ddots & \\
& & -r_{1}-r_{2} & 1+2 r_{1}-r_{3} & -r_{1}+r_{2} \\
& & & -r_{1}-r_{2} & 1+2 r_{1}-r_{3}
\end{array}\right)_{l \times l} \\
& \left(\begin{array}{c}
u_{i_{0}+1}^{n+1} \\
u_{i_{0}+2}^{n+1} \\
\vdots \\
u_{i_{0}+l-1}^{n+1} \\
u_{i_{0}+l}^{n+1}
\end{array}\right)_{l \times 1} \\
& =\left(\begin{array}{c}
\left(r_{1}+r_{2}\right) u_{i_{0}}^{n+1}+u_{i_{0}+1}^{n} \\
u_{i_{0}+2}^{n} \\
\vdots \\
u_{i_{0}+l-1}^{n} \\
\left(r_{1}-r_{2}\right) u_{i_{0}+l+1}^{n+1}+u_{i_{0}+l}^{n}
\end{array}\right)_{l \times 1} \text {. }
\end{aligned}
$$

A complete calculation step of the PASE-I scheme is as follows: $t^{\prime}$ is the number of time layers, $i^{\prime}$ is the number of segments, and $\bar{u}_{i^{\prime}}^{n+1}$ is the point on segment $i^{\prime}$ :

(1) for $t^{\prime}=1: N$

(2) if $\bmod \left(t^{\prime}, 2\right)=1$

(3) for $i^{\prime}=1: J$

(4) if $\bmod \left(i^{\prime}, 2\right)=1$

(5) solve equation (9) to get $\bar{u}_{i^{\prime}}^{n+1}$;

(6) else

(7) solve equation (10) to get $\bar{u}_{i^{\prime}}^{n+1}$;

(8) end

(9) end

(10) else

(11) for $i^{\prime}=1: J$

(12) $\quad$ if $\bmod \left(i^{\prime}, 2\right)=0$

(13) solve equation (9) to get $\bar{u}_{i^{\prime}}^{n+1}$;

(14) else

$$
\text { solve equation (10) to get } \bar{u}_{i^{\prime}}^{n+1} \text {; }
$$
end

(18) end

(19) end for

The matrix form of the PASE-I scheme is

$$
\left\{\begin{array}{l}
\left(I+G_{1}\right) U^{n+1}=\left(I-G_{2}\right) U^{n}+F_{1} \\
\left(I+G_{2}\right) U^{n+2}=\left(I-G_{1}\right) U^{n+1}+F_{2}
\end{array}\right.
$$

where

$$
\begin{aligned}
U^{n} & =\left(u_{1}^{n}, u_{2}^{n}, \ldots, u_{M-1}^{n}\right)^{T}, \\
F_{1} & =\left(\left(r_{1}+r_{2}\right) u_{0}^{n}, 0, \ldots, 0,\left(r_{1}-r_{2}\right) u_{M}^{n}\right)^{T}, \\
F_{2} & =\left(\left(r_{1}+r_{2}\right) u_{0}^{n+2}, 0, \ldots, 0,\left(r_{1}-r_{2}\right) u_{M}^{n+2}\right)^{T},
\end{aligned}
$$

in which $U^{n}, F_{1}$, and $F_{2}$ are the $(M-1)$ dimensional vectors. $A=-r_{1}-r_{2} ; B=-r_{1}+r_{2} ; Q_{l}$ is the (l)th-order zero matrix:

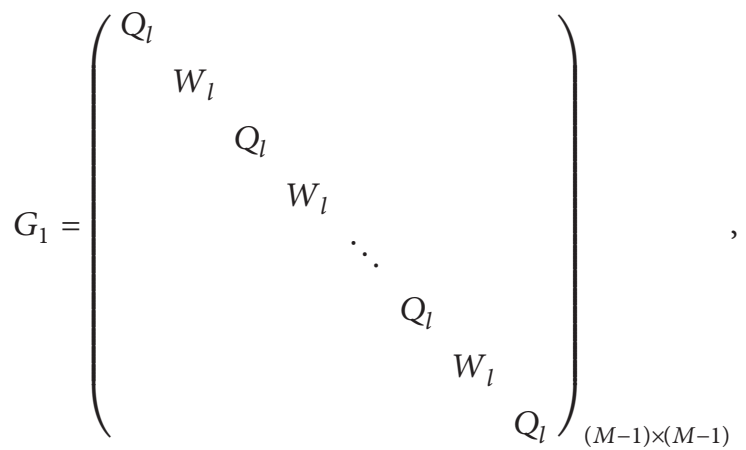

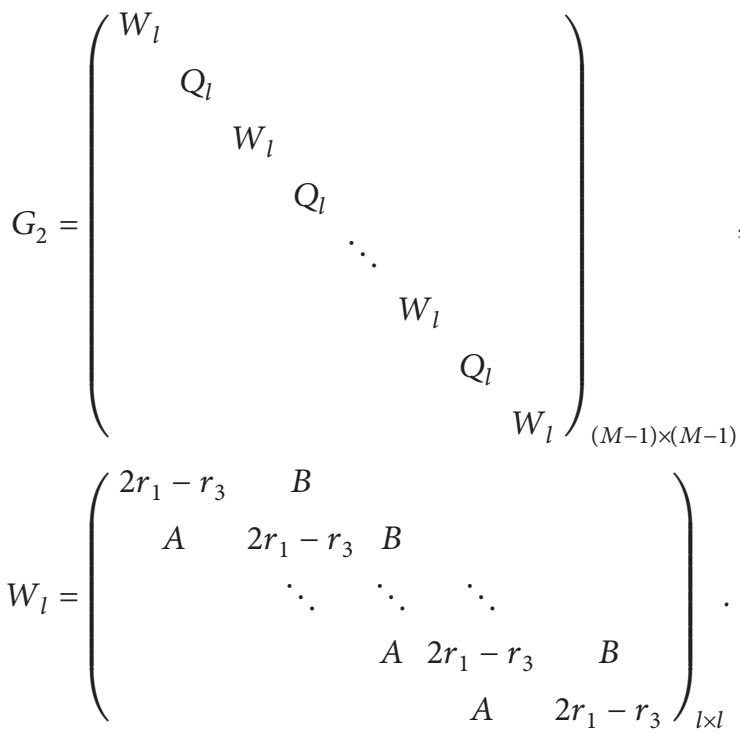

As can be seen from the piecewise pattern in Figure 1 and the expression of the matrix $G_{i}$, the PASE-I parallel difference scheme turns a discrete problem of orders $M-1$ into some small independent problems to be solved. The computation at each time level is only to solve a low-order equations system at each implicit segment. Compared with the classical implicit scheme, there are fewer $((J+1) / 2)$ equation systems in the odd layer and fewer $((J-1) / 2)$ 
equation systems in the even layer. The computation is simple, and the parallel characteristic is obvious.

\section{The Numerical Analysis of PASE-I Parallel Difference Method}

3.1. Existence and Uniqueness of Solution to PASE-I Scheme. In order to discuss the existence and the uniqueness of the PASE-I scheme solution, we need to introduce the following two lemmas.

Lemma 1 (Kellogg lemma, see [24]). Let $\theta>0$, and let $(G+$ $G^{T}$ ) be a nonnegative (or positive) definite matrix. Then, $(\theta I+G)^{-1}$ exists, and

$$
\left\|(\theta I+G)^{-1}\right\|_{2} \leq \theta^{-1} .
$$

Lemma 2. $G_{1}$ and $G_{2}$ in the PASE-I scheme (11) for solving the Burgers-Fisher equation are nonnegative definite matrices.

Proof. We only need to prove $G_{1}+\left(G_{1}\right)^{T}$ and $G_{2}+\left(G_{2}\right)^{T}$ are nonnegative definite matrices. That is to say, $W_{l}+\left(W_{l}\right)^{T}$ is a nonnegative definite matrix. The coefficient $a_{i}^{n}$ in formula (11) is assumed to be constant $a$, calculate as follows:

$$
\begin{aligned}
W_{l}+\left(W_{l}\right)^{T} & \\
= & \left(\begin{array}{ccccc}
4 r_{1}-2 r_{3} & A+B & & & \\
A+B & 4 r_{1}-2 r_{3} & A+B & & \\
& \ddots & \ddots & \ddots & \\
& & A+B & 4 r_{1}-2 r_{3} & A+B \\
& & & A+B & 4 r_{1}-2 r_{3}
\end{array}\right)_{l \times l} .
\end{aligned}
$$

We consider the case of low-speed flow and might as well set $0<u<1$. With $A=-r_{1}-r_{2}, B=-r_{1}+r_{2}, \quad r_{1}>r_{2}$, $r_{3}=\beta\left(1-a_{i}^{n}\right) \Delta t<0$, and $4 r_{1}-2 r_{3}>0, W_{l}+\left(W_{l}\right)^{T}$ is a diagonally dominant matrix, and the diagonal elements of $W_{l}+\left(W_{l}\right)^{T}$ are nonnegative real numbers. Therefore, $G_{1}+$ $\left(G_{1}\right)^{T}$ is a nonnegative definite matrix. Similarly, $G_{2}+\left(G_{2}\right)^{T}$ is also a nonnegative definite matrix. Therefore, $G_{1}$ and $G_{2}$ are nonnegative definite matrices.

From the initial conditions and the boundary conditions of the Burgers-Fisher equation, we know the value of $U^{0}$. Assuming that the value $U^{2 n}$ of the $(2 n)$ th time layer is known, the value $U^{2 n+1}$ of the $(2 n+1)$ th time layer waits for calculating. From the PASE-I scheme (11), the matrix equation for calculating the value of the $(2 n+1)$ th time layer is

$$
\left(I+G_{1}\right) U^{2 n+1}=\left(I-G_{2}\right) U^{2 n}+F_{1} .
$$

Apparently, the right of equation (16) is known, and $\left(I+G_{1}\right)^{-1}$ exists by Lemmas 1 and 2 . Then, equation (16) has a unique solution.

In the same way, applying the PASE-I scheme to calculate the value of the $(2 n+2)$ th time layer, the matrix equation is

$$
\left(I+G_{2}\right) U^{2 n+2}=\left(I-G_{1}\right) U^{2 n+1}+F_{2} .
$$

We could also prove that the matrix equation (17) has a unique solution. Then, we could get the following.

Theorem 1. The solution of the PASE-I parallel difference scheme (11) for the Burgers-Fisher equation is existing and unique.

\subsection{Linear Absolute Stability of PASE-I Scheme}

Lemma 3 (see [24]). Let $\theta>0$, and let $\left(G+G^{T}\right)$ be a nonnegative definite matrix. Then,

$$
\left\|(\theta I-G)(\theta I+G)^{-1}\right\|_{2} \leq 1 .
$$

In the following stability analysis, the coefficient $a_{i}^{n}$ in formula (11) is assumed to be constant $a$. By eliminating $U^{n+1}$, formula (11) can be rewritten as $U^{n}=G U^{n-2}$, where $G$ is the growth matrix and

$$
G=\left(I+G_{2}\right)^{-1}\left(I-G_{1}\right)\left(I+G_{1}\right)^{-1}\left(I-G_{2}\right) .
$$

For any even number $n$, there is

$$
\begin{aligned}
G^{n}= & \left(I+G_{2}\right)^{-1}\left(I-G_{1}\right)\left(I+G_{1}\right)^{-1}\left[\left(I-G_{2}\right)\right. \\
& \left.\left(I+G_{2}\right)^{-1}\left(I-G_{1}\right)\left(I+G_{1}\right)^{-1}\right]^{n-1}\left(I-G_{2}\right) .
\end{aligned}
$$

From Lemmas $1-3$, for any $r_{1}, r_{2}$, and $r_{3}$, there is

$$
\begin{array}{r}
\left\|\left(I+G_{2}\right)^{-1}\right\|_{2} \leq 1, \\
\left\|\left(I-G_{i}\right)\left(I+G_{i}\right)^{-1}\right\|_{2} \leq 1, \\
\text { where } i \text { is } 1 \text { or } 2 .
\end{array}
$$

So,

$$
\begin{aligned}
\left\|G^{n}\right\|_{2} \leq & \left\|\left(I+G_{2}\right)^{-1}\right\|_{2}\left\|\left(I-G_{1}\right)\left(I+G_{1}\right)^{-1}\right\|_{2}^{n} \\
& \cdot\left\|\left(I-G_{2}\right)\left(I+G_{2}\right)^{-1}\right\|_{2}^{n-1}\left\|\left(I-G_{2}\right)\right\|_{2} \\
\leq & \left\|\left(I-G_{2}\right)\right\|_{2} .
\end{aligned}
$$

We can get the following inequality easily:

$$
\begin{gathered}
\left\|\left(I-G_{2}\right)\right\|_{\infty} \leq 1+4 r_{1}+2 r_{2}-r_{3}, \\
\left\|\left(I-G_{2}\right)\right\|_{1} \leq 1+4 r_{1}+2 r_{2}-r_{3},
\end{gathered}
$$

where $r_{3}<0$.

Therefore

$$
\left\|\left(I-G_{2}\right)\right\|_{2} \leq \sqrt{\left\|\left(I-G_{2}\right)\right\|_{\infty}\left\|\left(I-G_{2}\right)\right\|_{1}} \leq C,
$$

where $C=1+4 r_{1}+2 r_{2}-r_{3}$. Then, there is

$$
\left\|G^{n}\right\|_{2} \leq C \text {. }
$$

Therefore, we have the following theorem.

Theorem 2. The PASE-I parallel difference scheme (11) for the Burgers-Fisher equation is linearly absolute stable. 
3.3. Computational Accuracy of PASE-I Scheme. The classical explicit scheme is

$$
\begin{aligned}
& \frac{u_{i}^{n+1}-u_{i}^{n}}{\Delta t}+\alpha u^{\delta} \frac{u_{i+1}^{n}-u_{i-1}^{n}}{2 \Delta x} \\
& =\mu \frac{u_{i+1}^{n}-2 u_{i}^{n}+u_{i-1}^{n}}{(\Delta x)^{2}}+\beta u_{i}^{n}\left(1-u^{\delta}\right) .
\end{aligned}
$$

The classical implicit scheme is

$$
\begin{aligned}
& \frac{u_{i}^{n+2}-u_{i}^{n+1}}{\Delta t}+\alpha u^{\delta} \frac{u_{i+1}^{n+2}-u_{i-1}^{n+2}}{2 \Delta x} \\
& =\mu \frac{u_{i+1}^{n+2}-2 u_{i}^{n+2}+u_{i-1}^{n+2}}{(\Delta x)^{2}}+\beta u_{i}^{n+2}\left(1-u^{\delta}\right) .
\end{aligned}
$$

Let each point of the above two schemes be expanded as the Taylor series at the point $u_{i}^{n+1}$. The truncation errors are, respectively, $T_{1}(\Delta t, \Delta x)$ and $T_{2}(\Delta t, \Delta x)$. Then, we get

$$
\begin{aligned}
T_{1}(\Delta t, \Delta x)= & \left(\frac{\partial u}{\partial t}-\frac{\Delta t}{2} \frac{\partial^{2} u}{\partial t^{2}}+\frac{\Delta t^{2}}{6} \frac{\partial^{3} u}{\partial t^{3}}\right)+\alpha u^{\delta}\left(\frac{\partial u}{\partial x}-\Delta t \frac{\partial^{2} u}{\partial x \partial t}+\frac{\Delta x^{2}}{6} \frac{\partial^{3} u}{\partial x^{3}}+\frac{\Delta t^{2}}{2} \frac{\partial^{3} u}{\partial x \partial t^{2}}\right. \\
& \left.-\frac{\Delta x^{2} \Delta t}{6} \frac{\partial^{4} u}{\partial x^{3} \partial t}-\frac{\Delta t^{3}}{6} \frac{\partial^{4} u}{\partial x \partial t^{3}}\right)-\mu\left(\frac{\partial^{2} u}{\partial x^{2}}-\Delta t \frac{\partial^{3} u}{\partial x^{2} \partial t}+\frac{\Delta x^{2}}{12} \frac{\partial^{4} u}{\partial x^{4}}+\frac{\Delta t^{2}}{2} \frac{\partial^{4} u}{\partial x^{2} \partial t^{2}}\right) \\
& -\beta\left(1-u^{\delta}\right)\left(u_{i}^{n+1}-\Delta t \frac{\partial u}{\partial t}+\frac{\Delta t^{2}}{2} \frac{\partial^{2} u}{\partial t^{2}}-\frac{\Delta t^{3}}{6} \frac{\partial^{3} u}{\partial t^{3}}\right)+O\left(\Delta x^{a} \Delta t^{b}\right), \\
T_{2}(\Delta t, \Delta x)= & \left(\frac{\partial u}{\partial t}+\frac{\Delta t}{2} \frac{\partial^{2} u}{\partial t^{2}}+\frac{\Delta t^{2}}{6} \frac{\partial^{3} u}{\partial t^{3}}\right)+\alpha u^{\delta}\left(\frac{\partial u}{\partial x}+\Delta t \frac{\partial^{2} u}{\partial x \partial t}+\frac{\Delta x^{2}}{6} \frac{\partial^{3} u}{\partial x^{3}}+\frac{\Delta t^{2}}{2} \frac{\partial^{3} u}{\partial x \partial t^{2}}\right. \\
& \left.+\frac{\Delta x^{2} \Delta t}{6} \frac{\partial^{4} u}{\partial x^{3} \partial t}+\frac{\Delta t^{3}}{6} \frac{\partial^{4} u}{\partial x \partial t^{3}}\right)-\mu\left(\frac{\partial^{2} u}{\partial x^{2}}+\Delta t \frac{\partial^{3} u}{\partial x^{2} \partial t}+\frac{\Delta x^{2}}{12} \frac{\partial^{4} u}{\partial x^{4}}+\frac{\Delta t^{2}}{2} \frac{\partial^{4} u}{\partial x^{2} \partial t^{2}}\right) \\
& -\beta\left(1-u^{\delta}\right)\left(u_{i}^{n+1}+\Delta t \frac{\partial u}{\partial t}+\frac{\Delta t^{2}}{2} \frac{\partial^{2} u}{\partial t^{2}}+\frac{\Delta t^{3}}{6} \frac{\partial^{3} u}{\partial t^{3}}\right)+O\left(\Delta x^{a} \Delta t^{b}\right),
\end{aligned}
$$

where $O\left(\Delta x^{a} \Delta t^{b}\right)=O\left(\Delta t+\Delta x^{2} \Delta t+\Delta x^{2}\right)$.

With the equation $(\partial u / \partial t)+\alpha u^{\delta}(\partial u / \partial x)-\mu\left(\partial^{2} u /\right.$ $\left.\partial x^{2}\right)-\beta u\left(1-u^{\delta}\right)=0$, we can get

$$
\begin{aligned}
T_{1}(\Delta t, \Delta x)= & \left(-\frac{\Delta t}{2} \frac{\partial^{2} u}{\partial t^{2}}+\frac{\Delta t^{2}}{6} \frac{\partial^{3} u}{\partial t^{3}}\right)+\alpha u^{\delta}\left(-\Delta t \frac{\partial^{2} u}{\partial x \partial t}+\frac{\Delta x^{2}}{6} \frac{\partial^{3} u}{\partial x^{3}}+\frac{\Delta t^{2}}{2} \frac{\partial^{3} u}{\partial x \partial t^{2}}\right. \\
& \left.-\frac{\Delta x^{2} \Delta t}{6} \frac{\partial^{4} u}{\partial x^{3} \partial t}-\frac{\Delta t^{3}}{6} \frac{\partial^{4} u}{\partial x \partial t^{3}}\right)-\mu\left(-\Delta t \frac{\partial^{3} u}{\partial x^{2} \partial t}+\frac{\Delta x^{2}}{12} \frac{\partial^{4} u}{\partial x^{4}}+\frac{\Delta t^{2}}{2} \frac{\partial^{4} u}{\partial x^{2} \partial t^{2}}\right) \\
& -\beta\left(1-u^{\delta}\right)\left(-\Delta t \frac{\partial u}{\partial t}+\frac{\Delta t^{2}}{2} \frac{\partial^{2} u}{\partial t^{2}}-\frac{\Delta t^{3}}{6} \frac{\partial^{3} u}{\partial t^{3}}\right)+O\left(\Delta x^{a} \Delta t^{b}\right), \\
T_{2}(\Delta t, \Delta x)= & \left(\frac{\Delta t}{2} \frac{\partial^{2} u}{\partial t^{2}}+\frac{\Delta t^{2}}{6} \frac{\partial^{3} u}{\partial t^{3}}\right)+\alpha u^{\delta}\left(\Delta t \frac{\partial^{2} u}{\partial x \partial t}+\frac{\Delta x^{2}}{6} \frac{\partial^{3} u}{\partial x^{3}}+\frac{\Delta t^{2}}{2} \frac{\partial^{3} u}{\partial x \partial t^{2}}\right. \\
& \left.+\frac{\Delta x^{2} \Delta t}{6} \frac{\partial^{4} u}{\partial x^{3} \partial t}+\frac{\Delta t^{3}}{6} \frac{\partial^{4} u}{\partial x \partial t^{3}}\right)-\mu\left(\Delta t \frac{\partial^{3} u}{\partial x^{2} \partial t}+\frac{\Delta x^{2}}{12} \frac{\partial^{4} u}{\partial x^{4}}+\frac{\Delta t^{2}}{2} \frac{\partial^{4} u}{\partial x^{2} \partial t^{2}}\right) \\
& -\beta\left(1-u^{\delta}\right)\left(\Delta t \frac{\partial u}{\partial t}+\frac{\Delta t^{2}}{2} \frac{\partial^{2} u}{\partial t^{2}}+\frac{\Delta t^{3}}{6} \frac{\partial^{3} u}{\partial t^{3}}\right)+O\left(\Delta x^{a} \Delta t^{b}\right) .
\end{aligned}
$$


In the expressions of $T_{1}(\Delta t, \Delta x)$ and $T_{2}(\Delta t, \Delta x)$, the terms with the same absolute value but the opposite sign are contained, respectively. Piecewise explicit and piecewise implicit alternate not only at the same time level but also at different time levels. Some error terms will cancel each other. Therefore, the computational accuracy of the PASE-I scheme is $O\left(\Delta x^{2}+\Delta t^{2}\right)$.

Theorem 3. The computational accuracy of the PASE-I parallel difference scheme (11) for the Burgers-Fisher equation is $O\left(\Delta x^{2}+\Delta t^{2}\right)$.

3.4. Convergence of PASE-I Scheme. This section provides a method to linearize the nonlinear equations [25-27]. The nonlinear problem is as follows:

$$
\begin{aligned}
& -\mu \Delta t \frac{\partial^{2} u^{n+1}}{\partial x^{2}}+\alpha \Delta t u^{n+1} \frac{\partial u^{n+1}}{\partial x}-\beta \Delta t\left(1-u^{n+1}\right) u^{n+1} \\
& +u^{n+1}=u^{n}, \quad x \in \Omega_{x}=[0,1], n \geq 0, \\
& u^{0}=u(x, 0)=f(x), \quad x \in \bar{\Omega}_{x}, \\
& u^{n+1}(0)=g_{1}\left(t^{n+1}\right), u^{n+1}(1)=g_{2}\left(t^{n+1}\right), \quad n \geq 0 .
\end{aligned}
$$

Assume that $u_{(k)}(x)$ is the $k$ th nominal solution of problem (30). An application of the quasilinearization process to the nonlinear problem (30) introduces a sequence $\left\langle u_{(k)}\right\rangle_{k=0}^{\infty}$ of linear equations determined by the following recurrence relation:

$$
\begin{aligned}
& -\mu \Delta t \frac{\partial^{2} u_{(k+1)}^{n+1}}{\partial x^{2}}+\alpha \Delta t u_{(k)}^{n+1} \frac{\partial u_{(k+1)}^{n+1}}{\partial x} \\
& +\left(\alpha \Delta t u_{(k)}^{n+1} \frac{\partial u_{(k)}^{n+1}}{\partial x}+\beta \Delta t\left(u_{(k)}^{n+1}-\left(1-u_{(k)}^{n+1}\right) u_{(k)}^{n+1}\right.\right. \\
& \left.\left.-\left(1-u_{(k)}^{n+1}\right)\right)+1\right) u_{(k+1)}^{n+1} \\
= & u_{(k+1)}^{n}+\alpha \Delta t u_{(k)}^{n+1} \frac{\partial u_{(k)}^{n+1}}{\partial x} \\
& +\beta \Delta t\left(u_{(k)}^{n+1}-\left(1-u_{(k)}^{n+1}\right)\right), \quad x \in \Omega_{x}, n \geq 0, \\
& u_{(k+1)}^{0}=f(x), \quad x \in \bar{\Omega}_{x}, \quad \\
& u_{(k+1)}^{n+1}(0)=g_{1}\left(t^{n+1}\right), u_{(k+1)}^{n+1}(1)=g_{2}\left(t^{n+1}\right), \quad n \geq 0,
\end{aligned}
$$

where $k=0,1,2, \ldots$ is the iteration index. This is an application of the Newton-Raphson-Kantorovich approximation method in function space. We choose a reasonable initial guess $u_{(0)}(x)$ satisfying the initial condition $f(x)$. For the sake of convenience, we let $u_{(k+1)}=\bar{u}$. Therefore, the above equation leads to the following initial boundary value problem:

$$
\begin{aligned}
& -\mu \Delta t \frac{\partial^{2} \bar{u}^{n+1}}{\partial x^{2}}+a(x) \Delta t \frac{\partial \bar{u}^{n+1}}{\partial x}+(1+\Delta t b(x)) \bar{u}^{n+1} \\
& =\bar{u}^{n}+\Delta t c(x), \quad x \in \Omega_{x}, n \geq 0, \\
& \bar{u}^{0}=f(x), \quad x \in \bar{\Omega}_{x}, \\
& \bar{u}^{n+1}(0)=g_{1}\left(t^{n+1}\right), \bar{u}^{n+1}(1)=g_{2}\left(t^{n+1}\right), \quad n \geq 0,
\end{aligned}
$$

where

$$
\begin{aligned}
a(x)= & a_{(k)}\left(x, t^{n+1}\right)=\alpha u_{(k)}^{n+1}, \\
b(x)= & b_{(k)}\left(x, t^{n+1}\right)=\alpha u_{(k)}^{n+1} \frac{\partial u_{(k)}^{n+1}}{\partial x} \\
& +\beta\left(u_{(k)}^{n+1}-\left(1-u_{(k)}^{n+1}\right) u_{(k)}^{n+1}-\left(1-u_{(k)}^{n+1}\right)\right), \\
c(x)= & c_{(k)}\left(x, t^{n+1}\right)=\alpha u_{(k)}^{n+1} \frac{\partial u_{(k)}^{n+1}}{\partial x}+\beta\left(u_{(k)}^{n+1}-\left(1-u_{(k)}^{n+1}\right)\right) .
\end{aligned}
$$

Further, we assume that the functions $a(x), b(x)$, and $c(x)$ are sufficiently smooth functions in the spatial direction with

$$
\begin{array}{ll}
a(x) \geq \eta>0, & x \in \bar{\Omega}_{x}, \\
b(x) \geq \varepsilon>0, & x \in \bar{\Omega}_{x} .
\end{array}
$$

By using quasilinearization process, we get the linear boundary value problem (32) for the function $\bar{u}^{n+1}=u_{(k+1)}^{n+1}$. For the solution $u^{n+1}(x)$ of original nonlinear problem (30), we require that

$$
\lim _{k \longrightarrow \infty} u_{(k)}^{n+1}(x)=u^{n+1}(x), \quad x \in \bar{\Omega}_{x},
$$

whereas numerically, we require that

$$
\left|\bar{u}^{n+1}(x)-u_{(k)}^{n+1}(x)\right|<\nu, \quad x \in \bar{\Omega}_{x},
$$

where $v$ is the small prescribed value to terminate the computation. This is the requisite criterion for terminating the iteration, and the solution $\bar{u}^{n+1}(x)$ is used as the numerical solution of the nonlinear boundary value problem (30).

Let $\left\langle u_{(k)}^{n+1}\right\rangle_{k=0}^{\infty}$ be the sequence of PASE-I scheme solutions at $(n+1)$ th time level. To prove the convergence of the PASE-I scheme, we consider the following equation:

$$
\begin{aligned}
\mu \frac{\partial^{2} u^{n+1}}{\partial x^{2}} & =H\left(u^{n+1}\right), \quad x \in \Omega_{x}, n \geq 0, \\
u^{n+1}(0) & =g_{1}\left(t^{n+1}\right), \\
u^{n+1}(1) & =g_{2}\left(t^{n+1}\right), \\
n \geq 0, &
\end{aligned}
$$


where $H\left(u^{n+1}\right)=\left(\partial u^{n+1} / \partial t\right)+\alpha u^{n+1}\left(\partial u^{n+1} / \partial x\right)-\beta u^{n+1}$ $\left(1-u^{n+1}\right)$.

We assume $u_{(0)}^{n+1}$ to be the initial value. By using quasilinearization process and the PASE-I scheme, we obtain a sequence $\left\langle u_{(k)}^{n+1}\right\rangle_{k=0}^{\infty}$ of linear equations determined by the following recurrence relation:

$$
\begin{aligned}
\mu \frac{\partial^{2} u_{(k+1)}^{n+1}}{\partial x^{2}} \approx & H\left(u_{(k)}^{n+1}\right)+\left(u_{(k+1)}^{n+1}-u_{(k)}^{n+1}\right) \\
& \cdot \frac{\partial H}{\partial u_{(k)}^{n+1}}\left(u_{(k)}^{n+1}\right), \quad x \in \Omega_{x}, n \geq 0, \\
u_{(k+1)}^{n+1}(0)= & g_{1}\left(t^{n+1}\right), \\
u_{(k+1)}^{n+1}(1)= & g_{2}\left(t^{n+1}\right), n \geq 0 .
\end{aligned}
$$

Thus, we have

$$
\begin{aligned}
\mu\left(\frac{\partial^{2} u_{(k+2)}^{n+1}}{\partial x^{2}}-\frac{\partial^{2} u_{(k+1)}^{n+1}}{\partial x^{2}}\right)= & H\left(u_{(k+1)}^{n+1}\right)-H\left(u_{(k)}^{n+1}\right) \\
& -\left(u_{(k+1)}^{n+1}-u_{(k)}^{n+1}\right) \frac{\partial H}{\partial u_{(k)}^{n+1}}\left(u_{(k)}^{n+1}\right) \\
& +\left(u_{(k+2)}^{n+1}-u_{(k+1)}^{n+1}\right) \frac{\partial H}{\partial u_{(k+1)}^{n+1}}\left(u_{(k+1)}^{n+1}\right) .
\end{aligned}
$$

Converting it into an integral function by using Green's function, we have

$$
\begin{aligned}
\mu\left(u_{(k+2)}^{n+1}-u_{(k+1)}^{n+1}\right)= & \int_{0}^{1} G(x, s)\left[H\left(u_{(k+1)}^{n+1}\right)-H\left(u_{(k)}^{n+1}\right)\right. \\
& -\left(u_{(k+1)}^{n+1}-u_{(k)}^{n+1}\right) \frac{\partial H}{\partial u_{(k)}^{n+1}}\left(u_{(k)}^{n+1}\right) \\
& \left.+\left(u_{(k+2)}^{n+1}-u_{(k+1)}^{n+1}\right) \frac{\partial H}{\partial u_{(k+1)}^{n+1}}\left(u_{(k+1)}^{n+1}\right)\right] \mathrm{d} s,
\end{aligned}
$$

where Green's function $G(x, s)$ is defined by

$$
\begin{aligned}
G(x, s) & = \begin{cases}x(1-s), & 0 \leq x \leq s \leq 1, \\
(1-x) s, & 0 \leq s \leq x \leq 1,\end{cases} \\
\max _{x, s} G(x, s) & =\frac{1}{4} .
\end{aligned}
$$

The mean value theorem gives us

$$
\begin{aligned}
H\left(u_{(k+1)}^{n+1}\right)-H\left(u_{(k)}^{n+1}\right)= & \left(u_{(k+1)}^{n+1}-u_{(k)}^{n+1}\right) \frac{\partial H}{\partial u_{(k)}^{n+1}}\left(u_{(k)}^{n+1}\right) \\
& +\frac{\left(u_{(k+1)}^{n+1}-u_{(k)}^{n+1}\right)^{2}}{2} \frac{\partial^{2} H}{\partial\left(u^{n+1}\right)^{2}}(\theta),
\end{aligned}
$$

where $u_{(k)}^{n+1} \leq \theta \leq u_{(k+1)}^{n+1}$. Now, putting the value of $H\left(u_{(k+1)}^{n+1}\right)-$ $H\left(u_{(k)}^{n+1}\right)$ in equation (40), we get the following estimate:

$$
\begin{aligned}
\mu\left(u_{(k+2)}^{n+1}-u_{(k+1)}^{n+1}\right)= & \int_{0}^{1} G(x, s)\left[\frac{\left(u_{(k+1)}^{n+1}-u_{(k)}^{n+1}\right)^{2}}{2} \frac{\partial^{2} H}{\partial\left(u^{n+1}\right)^{2}}(\theta)\right. \\
& \left.+\left(u_{(k+2)}^{n+1}-u_{(k+1)}^{n+1}\right) \frac{\partial H}{\partial u_{(k+1)}^{n+1}}\left(u_{(k+1)}^{n+1}\right)\right] \mathrm{d} s .
\end{aligned}
$$

Let

$$
\begin{gathered}
\max _{\left|u^{n+1}\right| \leq 1}\left|\frac{\partial^{2} H}{\partial\left(u^{n+1}\right)^{2}}\left(u^{n+1}\right)\right|=p \\
\max _{\left|u^{n+1}\right| \leq 1}\left|\frac{\partial H}{\partial u^{n+1}}\left(u^{n+1}\right)\right|=q .
\end{gathered}
$$

So, we obtain

$$
\begin{aligned}
& \left|u_{(k+2)}^{n+1}-u_{(k+1)}^{n+1}\right| \\
& \quad \leq \frac{1}{4 \mu} \int_{0}^{1}\left\{\frac{p}{2}\left(u_{(k+1)}^{n+1}-u_{(k)}^{n+1}\right)^{2}+q\left|u_{(k+2)}^{n+1}-u_{(k+1)}^{n+1}\right|\right\} \mathrm{d} s .
\end{aligned}
$$

Taking the maximum norm over the spatial domain and after some simplification, we have

$$
\begin{aligned}
\left\|u_{(k+2)}^{n+1}-u_{(k+1)}^{n+1}\right\|_{\bar{\Omega}_{x}} & \leq \frac{p}{(8 \mu-2 q)}\left\|u_{(k+1)}^{n+1}-u_{(k)}^{n+1}\right\|_{\bar{\Omega}_{x}}^{2} \\
& \leq C\left\|u_{(k+1)}^{n+1}-u_{(k)}^{n+1}\right\|_{\bar{\Omega}_{x}}^{2} .
\end{aligned}
$$

Therefore, Theorem 4 is proved. The PASE-I scheme (11) for the Burgers-Fisher equation is convergent.

Theorem 4. Let $\left\langle u_{(k)}^{n+1}\right\rangle_{k=0}^{\infty}$ be the sequence of PASE-I scheme solutions at $(n+1)$ th time level. Then, there exists a constant $C>0$, independent of $k$, such that

$$
\left\|u_{(k+1)}^{n+1}-u_{(k)}^{n+1}\right\|_{\bar{\Omega}_{x}} \leq C\left\|u_{(k)}^{n+1}-u_{(k-1)}^{n+1}\right\|_{\bar{\Omega}_{x}}^{2},
$$

i.e., the PASE-I scheme (11) for the Burgers-Fisher equation is convergent.

\section{The PASI-E Parallel Difference Scheme for Burgers-Fisher Equation}

Similar to the process of constructing the PASE-I parallel difference scheme, we can also construct the PASI-E parallel difference scheme of the Burgers-Fisher equation.

When solving the odd layer, we can calculate according to the rule of "classical implicit-classical explicit-classical implicit." On the even time layer, the rule of calculation becomes "classical explicit-classical implicit-classical explicit." We then get the following PASI-E parallel difference scheme for the Burgers-Fisher equation: 


$$
\left\{\begin{array}{l}
\left(I+G_{2}\right) U^{n+1}=\left(I-G_{1}\right) U^{n}+F_{3} \\
\left(I+G_{1}\right) U^{n+2}=\left(I-G_{2}\right) U^{n+1}+F_{4}
\end{array}\right.
$$

with $F_{3}=F_{4}=\left(\left(r_{1}+r_{2}\right) u_{0}^{n+1}, 0, \ldots, 0,\left(r_{1}-r_{2}\right) u_{M}^{n+1}\right)^{T}$.

Imitating the numerical analysis process of the PASE-I parallel difference scheme, the following theorem is obtained.

Theorem 5. The pure alternating segment implicit-explicit (PASI-E) parallel difference scheme (48) for the BurgersFisher equation is uniquely solvable, linearly absolute stable, and convergent, besides that the order of convergence is $O\left(\Delta x^{2}+\Delta t^{2}\right)$

\section{Numerical Experiments}

Numerical experiments were done in MATLAB R2014a, based on the Intel Core i5-4200 CPU@2.50 GHz.

Example 1. Let $\delta=1, \alpha=0.1, \mu=1$, and $\beta=-0.25$. We consider the Burgers-Fisher equation as follows [28, 29]:

$$
\frac{\partial u}{\partial t}+\alpha u^{\delta} \frac{\partial u}{\partial x}=\mu \frac{\partial^{2} u}{\partial x^{2}}+\beta u\left(1-u^{\delta}\right), \quad 0 \leq x \leq 1,0 \leq t \leq 1,
$$

with the initial and boundary conditions

$$
\begin{aligned}
& u(x, 0)=\left[\frac{1}{2}+\frac{1}{2} \tanh \left(\frac{-\alpha \delta x}{2(1+\delta)}\right)\right]^{1 / \delta} \\
& u(0, t)=\left[\frac{1}{2}+\frac{1}{2} \tanh \left(\frac{-\alpha \delta}{2(1+\delta)}\left(-\left(\frac{\alpha}{1+\delta}+\frac{\beta(1+\delta)}{\alpha}\right) t\right)\right)\right]^{1 / \delta}, \\
& u(1, t)=\left[\frac{1}{2}+\frac{1}{2} \tanh \left(\frac{-\alpha \delta}{2(1+\delta)}\left(1-\left(\frac{\alpha}{1+\delta}+\frac{\beta(1+\delta)}{\alpha}\right) t\right)\right)\right]^{1 / \delta},
\end{aligned}
$$

and the analytic solution

$$
u(x, t)=\left[\frac{1}{2}+\frac{1}{2} \tanh \left(\frac{-\alpha \delta}{2(1+\delta)}\left(x-\left(\frac{\alpha}{1+\delta}+\frac{\beta(1+\delta)}{\alpha}\right) t\right)\right)\right]^{1 / \delta}
$$

By taking time layer $N=500$, space layer $M=501$, piecewise number $J=5$, interior point number $l=((M-1) / J)=100$, and parameter $\delta=1$, the analytic solution surface, implicit scheme solution surface, PASE-I scheme solution surface, and PASI-E scheme solution surface are given. Figures 2-5 show that these surfaces are of the same shape as a whole.

We take time layer $N=500$, space layer $M=501$, and parameter $\delta=1$. At $t=0.5$, the numerical solutions of the PASE-I scheme and the PASI-E scheme in this paper are compared with the analytic solution and the implicit scheme solution. The computing results are listed in Table 1 . It is clearly shown that the numerical solutions of the PASE-I scheme and the PASI-E scheme are very close to the analytic solution. The value of $u$ is between 0 and 1 , which is appropriate and is consistent with the theoretical analysis.

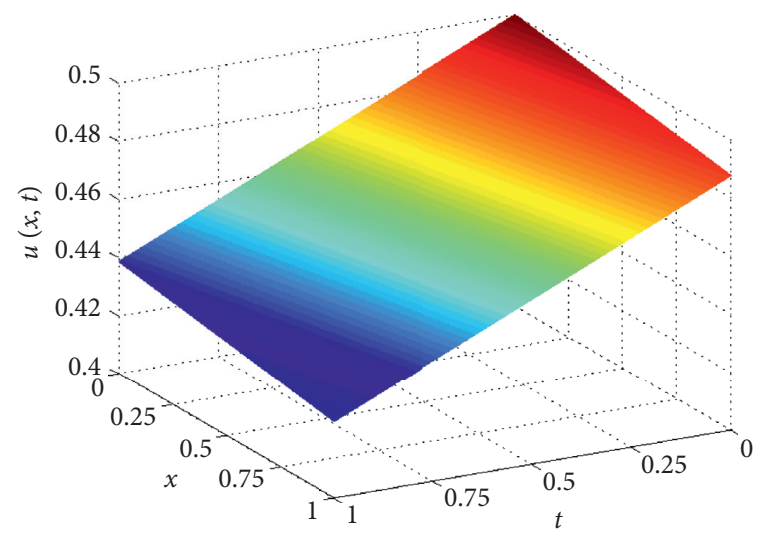

Figure 2: Curved surface of the analytic solution.

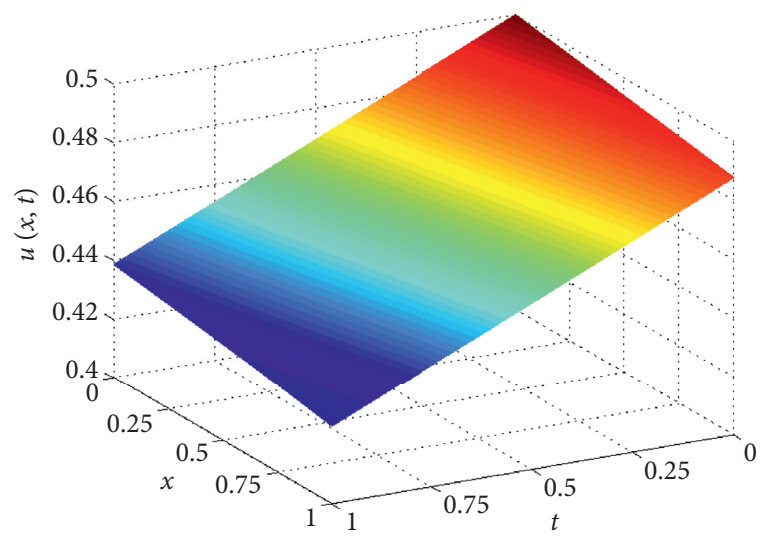

FIgURE 3: Curved surface of the implicit scheme solution.

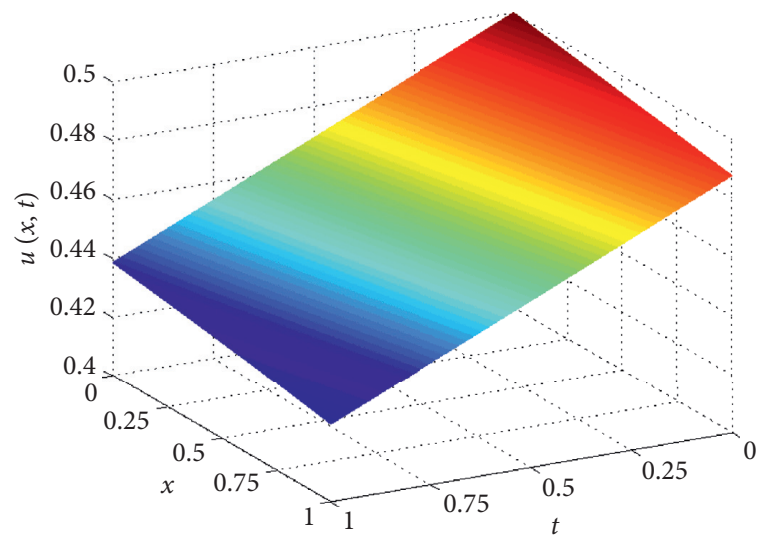

Figure 4: Curved surface of the PASE-I scheme solution.

Figure 6 is the comparison of the PASE-I scheme solution, PASI-E scheme solution, and analytic solution. Obviously, the solution curves of these two schemes are very close to the analytic solution. Therefore, the PASE-I and PASI-E parallel difference schemes are high-accuracy difference schemes for solving the Burgers-Fisher equation.

We treat the analytic solution $U_{i}^{j}$ as the control solution and the solution $\bar{U}_{i}^{j}$ of the PASE-I (PASI-E) scheme as the perturbation solution. The definition of the node error (NE) is $\mathrm{NE}=\bar{U}_{i}^{j}-U_{i}^{j}$. The node error distributions of the 


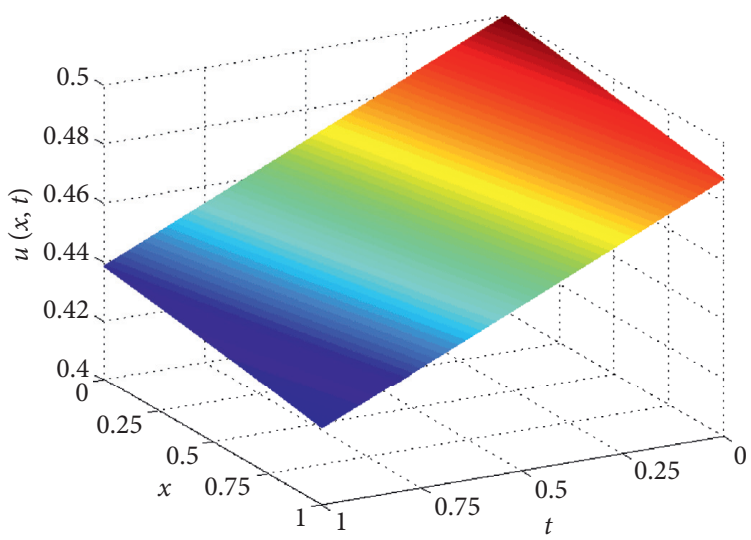

FIgURE 5: Curved surface of the PASI-E scheme solution.

TABLE 1: Comparison of numerical solutions and the analytic solution.

\begin{tabular}{lcccc}
\hline$x$ & Analytic solution & Implicit scheme solution & PASE-I scheme solution & PASI-E scheme solution \\
\hline 0.1018 & 0.467835 & 0.467835 & 0.467834 & 0.467834 \\
0.2016 & 0.466592 & 0.466593 & 0.466592 & 0.466592 \\
0.3014 & 0.465351 & 0.465354 & 0.465352 & 0.465352 \\
0.4012 & 0.464109 & 0.464117 & 0.464115 & 0.464115 \\
0.5010 & 0.462869 & 0.462882 & 0.462879 & 0.462879 \\
0.6008 & 0.461628 & 0.461647 & 0.461645 & 0.461645 \\
0.7006 & 0.460388 & 0.460412 & 0.460410 & 0.460410 \\
0.8004 & 0.459149 & 0.459173 & 0.459171 & 0.459171 \\
0.9002 & 0.457910 & 0.457927 & 0.457927 & 0.457927 \\
1.0000 & 0.456671 & 0.456671 & 0.456671 & 0.456671 \\
\hline
\end{tabular}

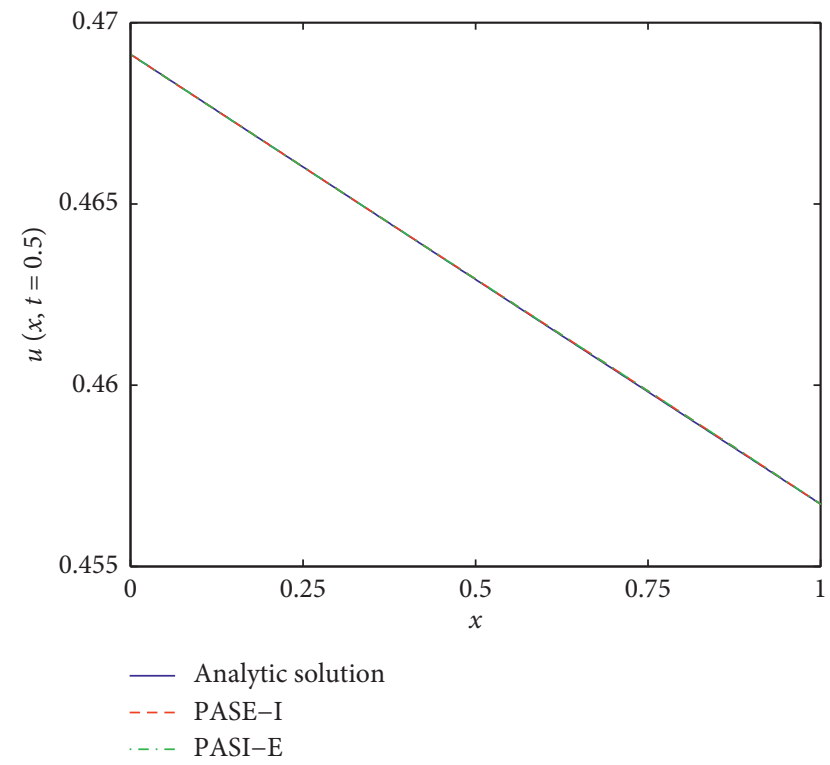

Figure 6: Comparison of PASE-I and PASI-E scheme solutions and the analytic solution.

implicit scheme solution, PASE-I scheme solution, and PASI-E scheme solution relative to the analytic solution are given below by taking time layer $N=500$, space layer $M=501$, and parameter $\delta=1$.
Figures 7-9 are, respectively, the node error distributions of the implicit scheme solution, PASE-I scheme solution, and PASI-E scheme solution relative to the analytic solution. As can be seen from these figures, the computing errors of the PASE-I scheme and the PASI-E scheme are similar to that of the implicit scheme, and the maximum error is less than $6 e-04$.

To verify the stability of the PASE-I scheme and the PASI-E scheme, the relative errors over time are given below. The definition of the sum of the relative error for every time level (SRET) is

$$
\operatorname{SRET}(j)=\sum_{i=1}^{M} \frac{\left|\bar{U}_{i}^{j}-U_{i}^{j}\right|}{U_{i}^{j}}
$$

It can be seen from Figure 10 that when we take the parameter $\delta=1$, the time layer $N=500$, and the space layer $M=501$, the SRET of the two scheme solutions remains stable and bounded with the increase in the time step. It also shows that the PASE-I and PASI-E methods of the Burgers-Fisher equation are computational stable.

Numerical experiments on the space and time convergence orders of the C-N scheme and the PASE-I scheme are given below. We define $L^{2}$ as the error, order 1 as the spaceconvergent order, and order 2 as the time-convergent order $[30,31]$ : 


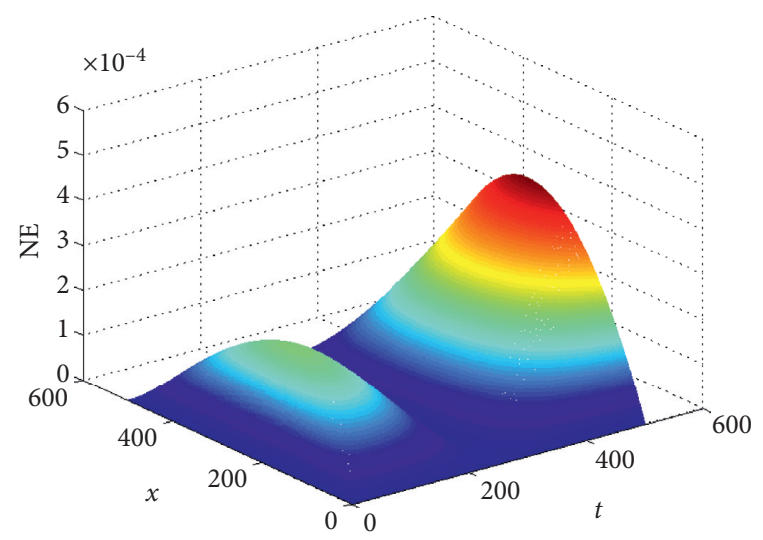

Figure 7: NE of the implicit scheme solution.

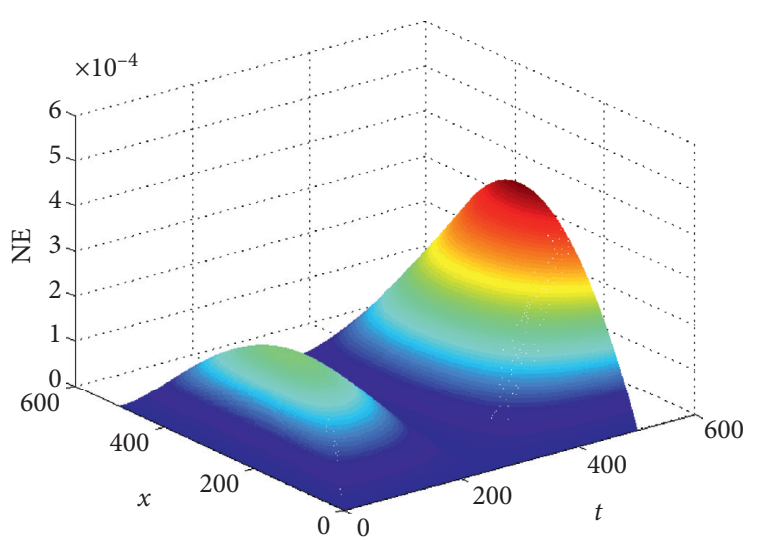

Figure 8: NE of the PASE-I scheme solution.

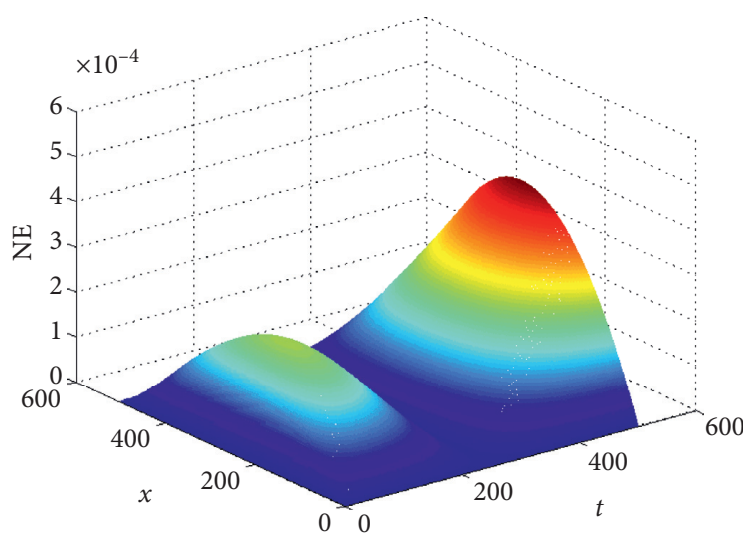

Figure 9: NE of the PASI-E scheme solution.

$$
\begin{aligned}
& L_{\Delta x}^{2}=\left\|U_{i}^{n}-{\overline{U_{i}}}^{n}\right\|^{2}=\left\{\sum_{i=1}^{m}\left(U_{i}^{n}-{\overline{U_{i}}}^{n}\right)^{2} \Delta x\right\}^{1 / 2}, \\
& L_{\Delta t}^{2}=\left\|U_{m}^{j}-{\overline{U_{m}}}^{j}\right\|^{2}=\left\{\sum_{j=1}^{n}\left(U_{m}^{j}-{\overline{U_{m}}}^{j}\right)^{2} \Delta t\right\}^{1 / 2},
\end{aligned}
$$

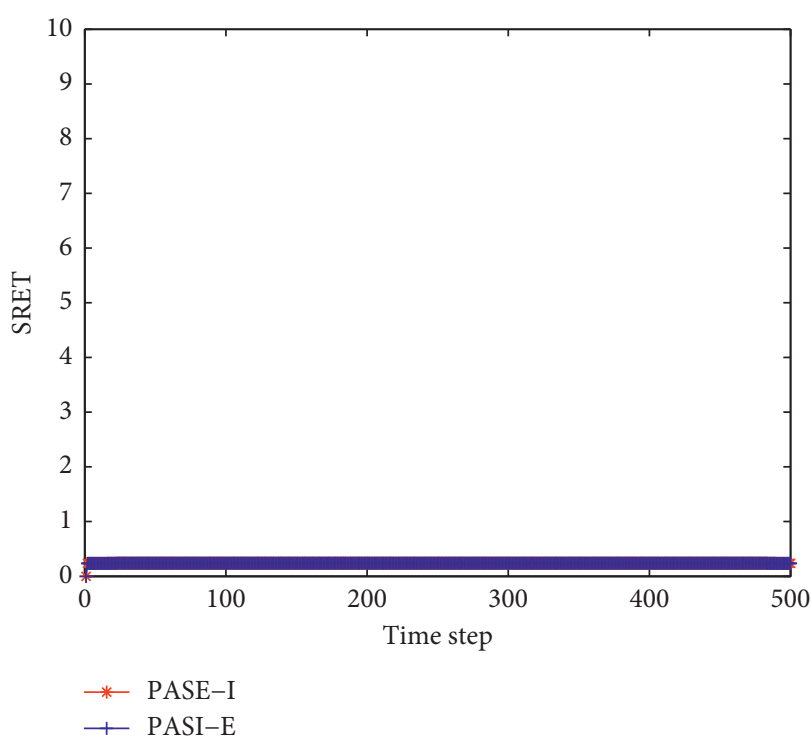

Figure 10: SRET of the PASE-I and PASI-E scheme solutions.

$$
\begin{aligned}
& \operatorname{order} 1=\frac{\log \left(L_{\Delta x_{1}}^{2} / L_{\Delta x_{2}}^{2}\right)}{\log \left(\Delta x_{1} / \Delta x_{2}\right)}, \\
& \operatorname{order} 2=\frac{\log \left(L_{\Delta t_{1}}^{2} / L_{\Delta t_{2}}^{2}\right)}{\log \left(\Delta t_{1} / \Delta t_{2}\right)} .
\end{aligned}
$$

At first, we verify the spatial accuracies of the C-N scheme and the PASE-I scheme by taking $M=21,41,81$, 161,321 and $N=\left(\left((M-1)^{2}\right) / 200\right)$. Table 2 shows that the spatial accuracies of the C-N scheme and the PASE-I scheme in this paper are approximately 2 .

Secondly, we calculate the time accuracies of the C-N scheme and the PASE-I scheme by taking $N=10,40,160$, 640,2560 . We set the space step $\Delta x=(1 / 451)$, that is, $M=451$.

Table 3 shows that the time accuracies of the C-N scheme and the PASE-I scheme are approximately 2 . Therefore, the PASE-I scheme constructed in this paper and the existing C$\mathrm{N}$ scheme possess the same order of spatial accuracy and time accuracy. The numerical results agree well with theoretical analysis.

In order to better verify the accuracies of the PASE-I method and the PASI-E method, the distribution of errors in spatial lattices is also investigated. We define difference total energy (DTE) as

$$
\operatorname{DTE}(i)=\frac{1}{2} \sum_{j=1}^{N}\left(\bar{U}_{i}^{j}-U_{i}^{j}\right)^{2} .
$$

As can be seen from Figure 11, when the time layer $N=$ 500 and the space layer $M=501$, the DTE of the PASE-I and PASI-E scheme solutions is between 0 and $1.2 e-05$. That is, the solutions of PASE-I and PASI-E schemes are very close to the analytic solution. Figure 11 shows that the PASE-I and PASI-E methods of the Burgers-Fisher equation have good accuracy. 
TABle 2: Numerical errors and space-convergent orders of two schemes.

\begin{tabular}{lcccc}
\hline & \multicolumn{2}{c}{ C-N scheme } & \multicolumn{2}{c}{ PASE-I scheme } \\
$M$ & $L_{\Delta x}^{2}$ & Order 1 & $L_{\Delta x}^{2}$ & Order 1 \\
\hline 21 & $6.156107 e-02$ & - & $6.507735 e-02$ & - \\
41 & $1.528761 e-02$ & 2.009655 & $1.737569 e-02$ & 1.905085 \\
81 & $3.812119 e-03$ & 2.003698 & $3.812119 e-03$ & 2.188406 \\
161 & $9.523667 e-04$ & 2.001004 & $9.523667 e-04$ & 2.001004 \\
321 & $2.380494 e-04$ & 2.000256 & $2.380494 e-04$ & 2.000256 \\
\hline
\end{tabular}

TABle 3: Numerical errors and time-convergent orders of two schemes.

\begin{tabular}{lcccc}
\hline & \multicolumn{2}{c}{ C-N scheme } & \multicolumn{2}{c}{ PASE-I scheme } \\
$N$ & $L_{\Delta t}^{2}$ & Order 2 & $L_{\Delta t}^{2}$ & Order 2 \\
\hline 10 & $5.545838 e-02$ & - & $5.843058 e-02$ & - \\
40 & $1.376361 e-02$ & 2.010546 & $1.452555 e-02$ & 2.008131 \\
160 & $3.431226 e-03$ & 2.004063 & $3.431226 e-03$ & 2.081797 \\
640 & $8.571503 e-04$ & 2.001104 & $8.571503 e-04$ & 2.001104 \\
2560 & $2.150054 e-04$ & 1.995175 & $2.148978 e-04$ & 1.995898 \\
\hline
\end{tabular}

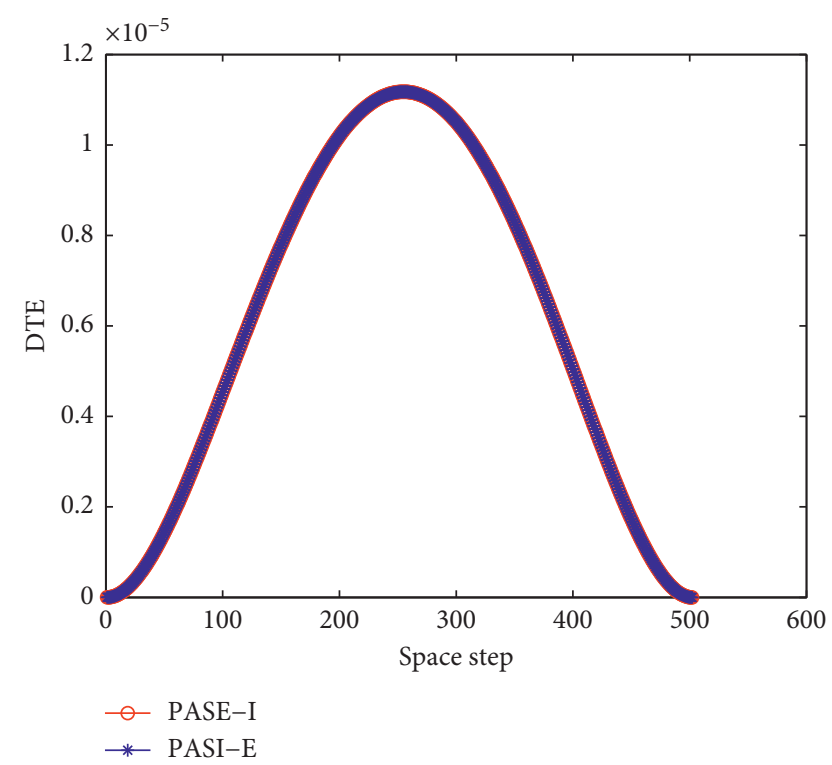

FIgure 11: DTE of the PASE-I and PASI-E scheme solutions.

The PASE-I scheme and the PASI-E scheme have superiority in the aspect of the computing time. But, this superiority can only be reflected when the amount of data is large. In case the number of grid points is less than a certain range, the effect of data communication on the cycle can reduce the computational efficiency, and the superiority of parallel computing is not obvious $[13,32]$. When the amount of data is large, the impact of program loop execution is much greater than that of data communication, and the parallel scheme is more effective.

Figure 12 shows that the computing time of three schemes increases with the increase in the number of space grid points. When the number of space grid points is greater

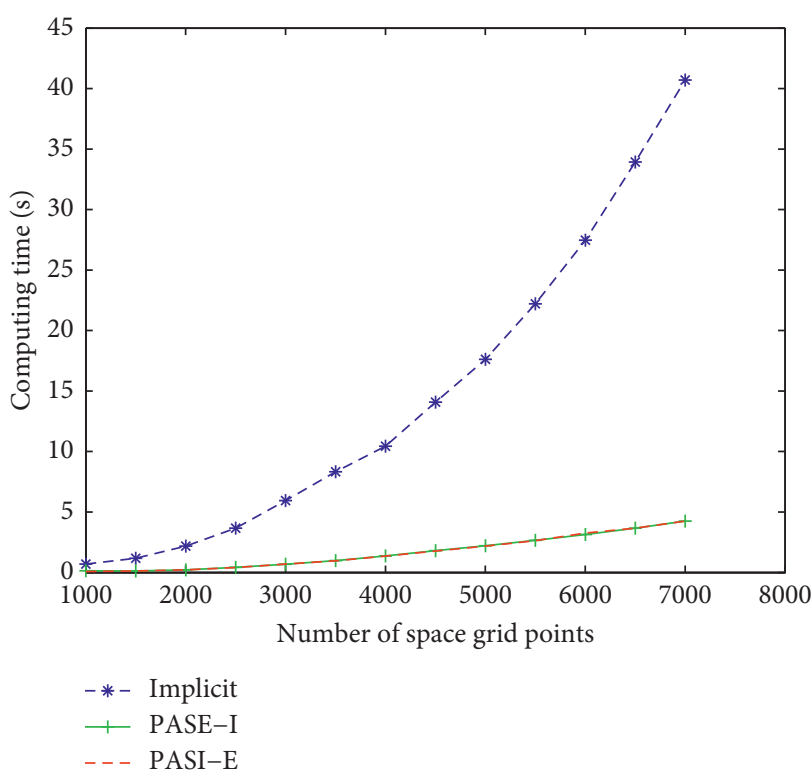

FIgURE 12: Comparison on the computing time of three schemes.

than 1000, the impact of program loop execution is much greater than that of data communication. The PASE-I scheme and the PASI-E scheme have obvious superiority with regard to the computing time.

To compare the computational efficiency of the implicit scheme, PASE-I scheme, and PASI-E scheme, the analysis of the scheme speedup ratio is done [33]. The speedup ratios of the PASE-I and PASI-E schemes relative to the implicit scheme are, respectively, $S_{p 1}$ and $S_{p 2}$. We fix the time layer as $N=500$ and take the number of space grids as $M=4001,4501,5001,5501,6001,6501$. The computational results are shown in Table 4 .

As we can see from Table 4, the computing time of three difference schemes is increasing with the increase in the number of space grids for the Burgers-Fisher equation. $S_{p 1}$ and $S_{p 2}$ are both greater than 7.6 and gradually increasing. It is shown that the PASE-I scheme and the PASI-E scheme have obvious advantages in saving the computing time. Therefore, the PASE-I scheme and the PASI-E scheme for the Burgers-Fisher equation are more efficient than the serial implicit scheme.

Example 2. Let $\delta=5, L=1$, and $T=1$. Consider the following Burgers-Fisher equation:

$$
\frac{\partial u}{\partial t}+\alpha u^{5} \frac{\partial u}{\partial x}=\mu \frac{\partial^{2} u}{\partial x^{2}}+\beta u\left(1-u^{5}\right) .
$$

The initial and boundary conditions are determined by the analytic solution, and the analytic solution is

$$
u(x, t)=\left[\frac{1}{2}+\frac{1}{2} \tanh \left(\frac{-5 \alpha}{12}\left(x-\left(\frac{\alpha}{6}+\frac{6 \beta}{\alpha}\right) t\right)\right)\right]^{1 / 5} .
$$

Figures 13 and 14 are, respectively, the node error distributions of the C-N scheme solution and the PASE-I scheme solution with respect to the analytic solution with the time layer $N=500$, the space layer $M=501$, and the 
TABLE 4: The computing time and speedup ratios of three schemes.

\begin{tabular}{lcccccc}
\hline Grid point & 4001 & 4501 & 5001 & 5501 & 6001 & 6501 \\
\hline Implicit & 10.439858 & 14.085181 & 17.624641 & 22.209831 & 27.466145 & 33.927893 \\
PASE-I & 1.370336 & 1.799631 & 2.210280 & 2.662593 & 3.135584 & 3.658886 \\
PASI-E & 1.358614 & 1.779123 & 2.174053 & 2.634832 & 3.256159 & 3.664002 \\
$S_{p 1}$ & 7.618466 & 7.826705 & 7.973940 & 8.341429 & 8.759499 & 9.272738 \\
$S_{p 2}$ & 7.684197 & 7.916924 & 8.106813 & 8.429316 & 8.435136 & 9.259791 \\
\hline
\end{tabular}

parameter $\delta=5$. As can be seen from Figures 13 and 14, the maximum errors will not exceed $3 e-03$, and the parameter $\delta$ has little effect on computational accuracy of the PASE-I scheme. It is shown that the PASE-I difference method with intrinsic parallelism in this paper is a high-precision difference method for solving the Burgers-Fisher equation.

The comparison and analysis of the computational efficiency of the two difference schemes are discussed as the spatial grid is continuously encrypted. For $N=500$, Figure 15 shows the comparison of computing time between the PASE-I scheme and the C-N scheme. We can see that the PASE-I scheme requires the computing time as the same as the $\mathrm{C}-\mathrm{N}$ scheme when $M<1000$. But, with the increase in the number of space grids, the computing time of the $\mathrm{C}-\mathrm{N}$ scheme increases exponentially, and the computing time of the PASE-I scheme increases linearly. Compared with the $\mathrm{C}-\mathrm{N}$ scheme, the advantage of the high efficiency of the PASE-I scheme is more and more obvious with the increase in the space grids.

The speedup $S_{p}=T_{1} / T_{p}$, and efficiency $E_{p}=S_{p} / p\left(T_{1}\right.$ is the computing time of $\mathrm{C}-\mathrm{N}$ and $T_{p}$ is the computing time of parallel scheme) are defined $[32,33]$. Using four cores for this numerical experiment, we fix the time layer as $N=500$ and take the number of space grid points as $M=2001,3001,4001,5001,6001,7001$. The computational results are shown in Table 5. Compared with the serial difference scheme, the computing time of the PASE-I parallel difference scheme increases slowly. $S_{p}$ is greater than 13.9 , and $E_{p}$ is greater than 3.4. The computational efficiency of the PASE-I parallel difference scheme is greatly improved with the refinement of the spatial mesh. When the number of space grid points is more than 2001, the computing time of the PASE-I scheme can save about $90 \%$ compared with the C-N scheme.

Respectively, taking $M=61,121,241,481,961$ and $N=$ $\left(\left((M-1)^{2}\right) / 300\right)$ and setting the space step $\Delta x=(1 / 501)$ and $N=10,40,160,640,2560$, Tables 6 and 7 show that the classical C-N scheme and the PASE-I scheme in this paper have an approximately second convergence rate in space direction and time direction when $\delta=5$.

Example 3. Let $L=1$ and $T=1$. The absolute error (AE) of the approximate solution of the Adomian decomposition method (ADM) [7] and the PASE-I scheme solution relative to the analytic solution under the three conditions in Tables $8-10$ is discussed.

From Tables $8-10$, we can know that when $\delta, \alpha$, and $\beta$ take different values, the PASE-I difference method has less error and can effectively solve the Burgers-Fisher equation.

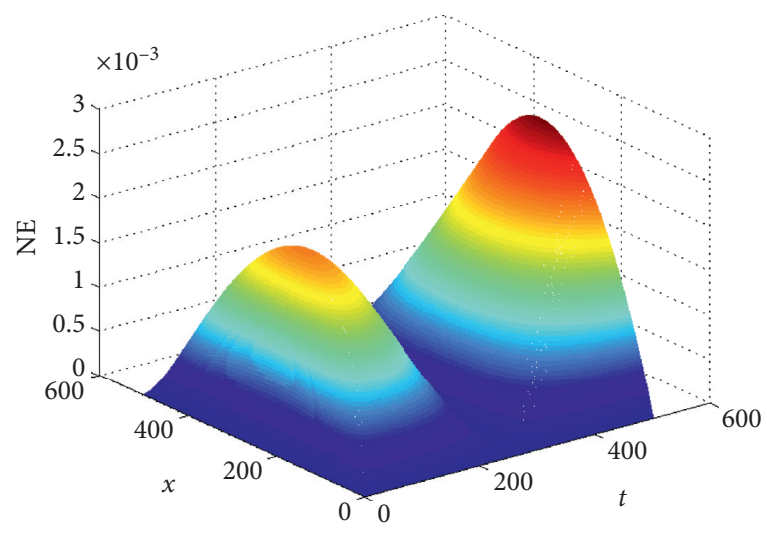

Figure 13: NE of the C-N scheme solution.

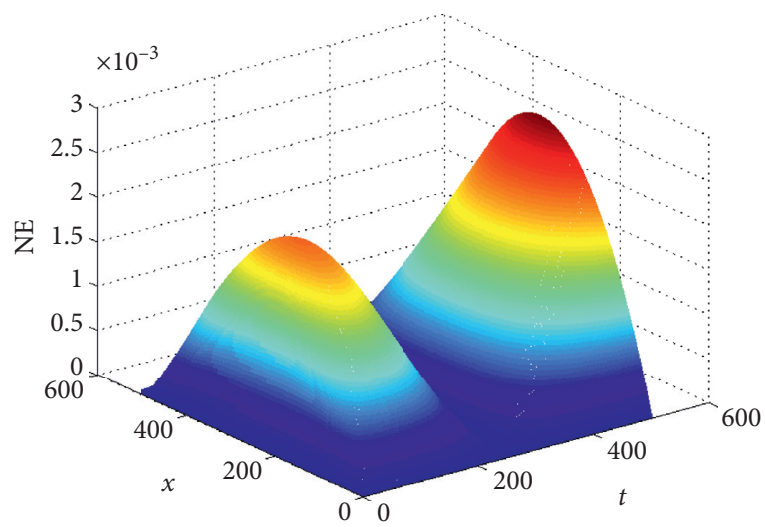

FIgURE 14: NE of the PASE-I scheme solution.

Example 4. Let $\delta=1, \alpha=0.1, \mu=1$, and $\beta=-0.25$. Consider the following Burgers-Fisher equation:

$$
\frac{\partial u}{\partial t}+\alpha u \frac{\partial u}{\partial x}=\mu \frac{\partial^{2} u}{\partial x^{2}}+\beta u\left(1-u^{\delta}\right), \quad 0 \leq x \leq 1,0 \leq t \leq 1,
$$

with the initial and boundary conditions

$$
\begin{aligned}
u(x, 0) & =0.45, \\
u(0, t)=u(1, t) & =0.45 .
\end{aligned}
$$

Let the numerical solution of the C-N scheme approximately substitute the analytic solution. Figure 16 is the comparison of the PASE-I scheme solution and the C-N scheme solution. Obviously, the numerical solution of the PASE-I scheme is very close to that of the C-N scheme. 


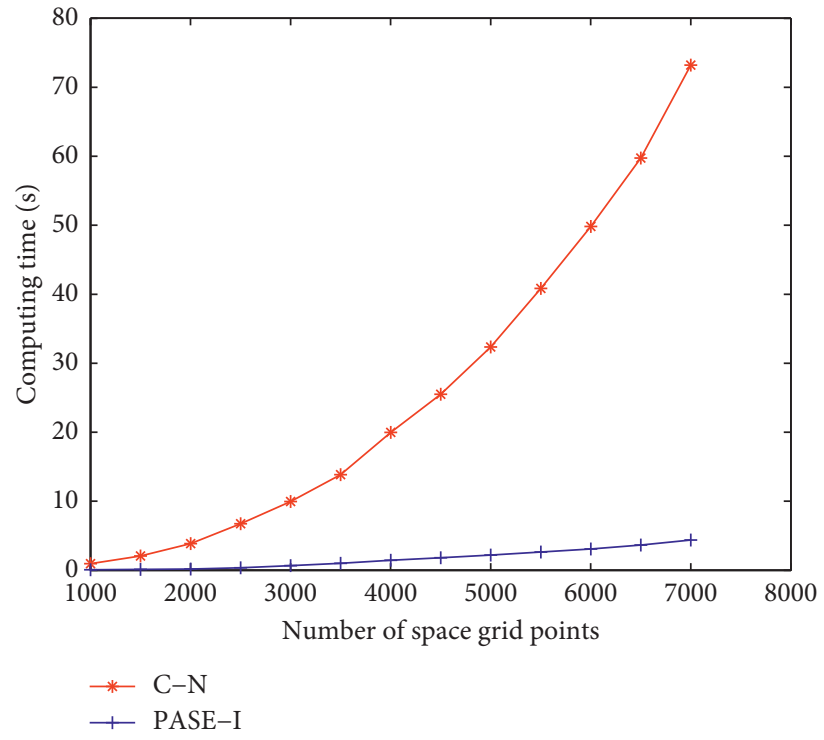

FIgURE 15: Comparison on the computing time of the C-N scheme and the PASE-I scheme.

TABle 5: The computing time, speedup, and efficiency of two schemes.

\begin{tabular}{|c|c|c|c|c|c|c|}
\hline Grid point & 2001 & 3001 & 4001 & 5001 & 6001 & 7001 \\
\hline $\mathrm{C}-\mathrm{N}$ & 3.847614 & 9.955498 & 19.975747 & 32.350496 & 49.821468 & 73.215463 \\
\hline PASE-I & 0.164309 & 0.668727 & 1.437007 & 2.197856 & 3.081143 & 4.377323 \\
\hline$S_{p}$ & 23.416940 & 14.887238 & 13.900939 & 14.719115 & 16.169801 & 16.726082 \\
\hline$\stackrel{p}{E_{p}}$ & 5.854235 & 3.721810 & 3.475235 & 3.679779 & 4.042450 & 4.181521 \\
\hline
\end{tabular}

TABLE 6: Numerical errors and space-convergent orders of two schemes.

\begin{tabular}{lcccc}
\hline & \multicolumn{2}{c}{ C-N scheme } & \multicolumn{2}{c}{ PASE-I scheme } \\
$M$ & $L_{\Delta x}^{2}$ & Order 1 & $L_{\Delta x}^{2}$ & Order 1 \\
\hline 61 & $2.375103 e-02$ & - & $2.479423 e-02$ & - \\
121 & $5.977703 e-03$ & 1.990327 & $5.965937 e-03$ & 2.055183 \\
241 & $1.496528 e-03$ & 1.997972 & $1.495851 e-03$ & 1.995783 \\
481 & $3.724303 e-04$ & 2.006577 & $3.724338 e-04$ & 2.005910 \\
961 & $9.270665 e-05$ & 2.006226 & $9.270631 e-05$ & 2.006245 \\
\hline
\end{tabular}

TABLE 7: Numerical errors and time-convergent orders of two schemes.

\begin{tabular}{lcccc}
\hline & \multicolumn{1}{c}{ C-N scheme } & \multicolumn{2}{c}{ PASE-I scheme } \\
$N$ & $L_{\Delta t}^{2}$ & Order 2 & $L_{\Delta t}^{2}$ & Order 2 \\
\hline 10 & $9.422589 e-02$ & - & $1.016623 e-01$ & 2.032326 \\
40 & $2.501432 e-02$ & 1.913369 & $2.485243 e-02$ & 1.986309 \\
160 & $6.283638 e-03$ & 1.993082 & $6.272349 e-03$ & 1.997225 \\
640 & $1.571107 e-03$ & 1.999819 & $1.571107 e-03$ & 2.056731 \\
2560 & $3.791313 e-04$ & 2.051012 & $3.776313 e-04$ & \\
\hline
\end{tabular}

From Figure 17, we see that when the number of grid points we need to calculate is greater than a certain range, the PASE-I scheme of this paper shows a clear superiority in computation time. With the increase in the grid number, the computing time of the two difference schemes rises for the Burgers-Fisher equation. But, the increased amplitude of the computing time of the $\mathrm{C}-\mathrm{N}$ scheme is greater than that of the PASE-I scheme, showing that the computing efficiency of the PASE-I scheme is great. 
TABLE 8: The absolute error for $\delta=1, \alpha=0.001$, and $\beta=0.001$.

\begin{tabular}{|c|c|c|c|c|c|c|}
\hline \multirow{2}{*}{$x$} & \multirow{2}{*}{$t$} & \multirow{2}{*}{ Analytic solution } & \multicolumn{2}{|c|}{$\mathrm{ADM}$} & \multicolumn{2}{|c|}{ PASE-I scheme } \\
\hline & & & Solution & $\mathrm{AE}$ & Solution & $\mathrm{AE}$ \\
\hline \multirow{3}{*}{0.1} & 0.005 & 0.499989 & 0.499979 & $9.68763 e-06$ & 0.499988 & $7.44768 e-07$ \\
\hline & 0.001 & 0.499987 & 0.499986 & $1.93753 e-06$ & 0.499987 & $3.50250 e-12$ \\
\hline & 0.010 & 0.499990 & 0.499971 & $1.93752 e-05$ & 0.499990 & $4.94485 e-07$ \\
\hline \multirow{3}{*}{0.5} & 0.005 & 0.499939 & 0.499929 & $9.68691 e-06$ & 0.499939 & $2.49842 e-07$ \\
\hline & 0.001 & 0.499938 & 0.499936 & $1.93738 e-06$ & 0.499938 & $2.12405 e-11$ \\
\hline & 0.010 & 0.499940 & 0.499921 & $1.93738 e-05$ & 0.499940 & $3.18600 e-10$ \\
\hline \multirow{3}{*}{0.9} & 0.005 & 0.499889 & 0.499879 & $9.68619 e-06$ & 0.499889 & $2.45126 e-07$ \\
\hline & 0.001 & 0.499888 & 0.499886 & $1.93724 e-06$ & 0.499888 & $4.59810 e-11$ \\
\hline & 0.010 & 0.499890 & 0.499871 & $1.93724 e-05$ & 0.499890 & $4.95285 e-07$ \\
\hline
\end{tabular}

TABLE 9: The absolute error for $\delta=2, \alpha=1$, and $\beta=1$.

\begin{tabular}{cccccrr}
\hline \multirow{2}{*}{$t$} & \multirow{2}{*}{$t$} & Analytic solution & Solution & ADM & \multicolumn{2}{c}{ PASE-I scheme } \\
& & & 0.694024 & $1.40177 e-03$ & 0.694903 & $5.22719 e-04$ \\
0.1 & 0.0005 & 0.695426 & 0.694986 & $2.80396 e-04$ & 0.694779 & $1.22063 e-05$ \\
& 0.0001 & 0.694792 & 0.692822 & $2.80301 e-03$ & 0.695055 & $5.69646 e-04$ \\
\hline \multirow{3}{*}{0.5} & 0.0010 & 0.695625 & 0.644952 & $1.34526 e-03$ & 0.646237 & $6.04821 e-05$ \\
& 0.0005 & 0.646297 & 0.645861 & $2.69094 e-04$ & 0.646118 & $1.18993 e-05$ \\
& 0.0001 & 0.646130 & 0.643816 & $2.69000 e-03$ & 0.646385 & $1.20878 e-04$ \\
\hline \multirow{3}{*}{0.9} & 0.0010 & 0.646506 & 0.594204 & $1.27699 e-03$ & 0.595920 & $4.38621 e-04$ \\
& 0.0005 & 0.595481 & 0.595055 & $2.55438 e-04$ & 0.595807 & $1.12264 e-05$ \\
& 0.0001 & 0.595818 & 0.593141 & $2.55346 e-03$ & 0.595431 & $3.69858 e-04$ \\
\hline
\end{tabular}

TABle 10: The absolute error for $\delta=3, \alpha=1$, and $\beta=0$.

\begin{tabular}{ccccccr}
\hline \multirow{2}{*}{$t$} & Analytic solution & \multicolumn{2}{c}{ ADM } & \multicolumn{2}{c}{ PASE-I scheme } \\
& & & Solution & AE & Solution & AE \\
\hline \multirow{3}{*}{0.1} & 0.0005 & 0.783267 & 0.784115 & $4.45320 e-04$ & 0.783283 & $1.53759 e-05$ \\
& 0.0001 & 0.783257 & 0.784106 & $4.46379 e-04$ & 0.783260 & $3.07691 e-06$ \\
& 0.0010 & 0.783280 & 0.784127 & $4.43997 e-04$ & 0.783310 & $3.06040 e-05$ \\
\hline \multirow{3}{*}{0.5} & 0.0005 & 0.741295 & 0.743150 & $1.85474 e-03$ & 0.741314 & $1.82655 e-05$ \\
& 0.0001 & 0.741284 & 0.743145 & $1.86057 e-03$ & 0.741288 & $3.65377 e-06$ \\
& 0.0010 & 0.741309 & 0.743157 & $1.84746 e-03$ & 0.741346 & $3.65239 e-05$ \\
\hline \multirow{3}{*}{0.9} & 0.0005 & 0.696625 & 0.697089 & $9.19582 e-04$ & 0.696646 & $2.06208 e-05$ \\
& 0.0001 & 0.696614 & 0.697089 & $9.31803 e-04$ & 0.696618 & $4.12657 e-06$ \\
& 0.0010 & 0.696640 & 0.697088 & $9.04297 e-04$ & 0.696681 & $4.10297 e-05$ \\
\hline
\end{tabular}

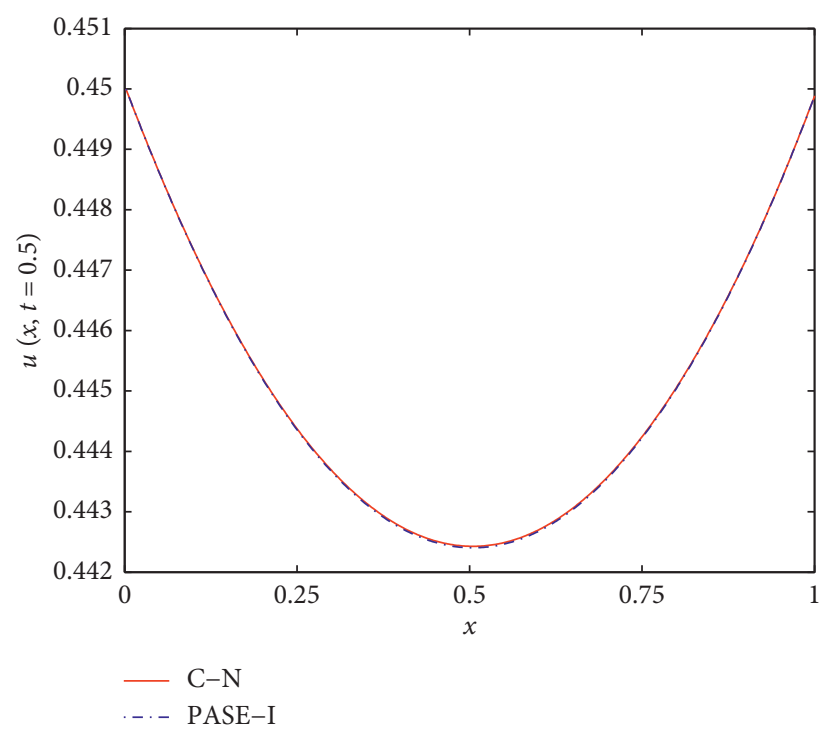

FIgURE 16: Comparison of the PASE-I scheme solution and the C-N scheme solution. 


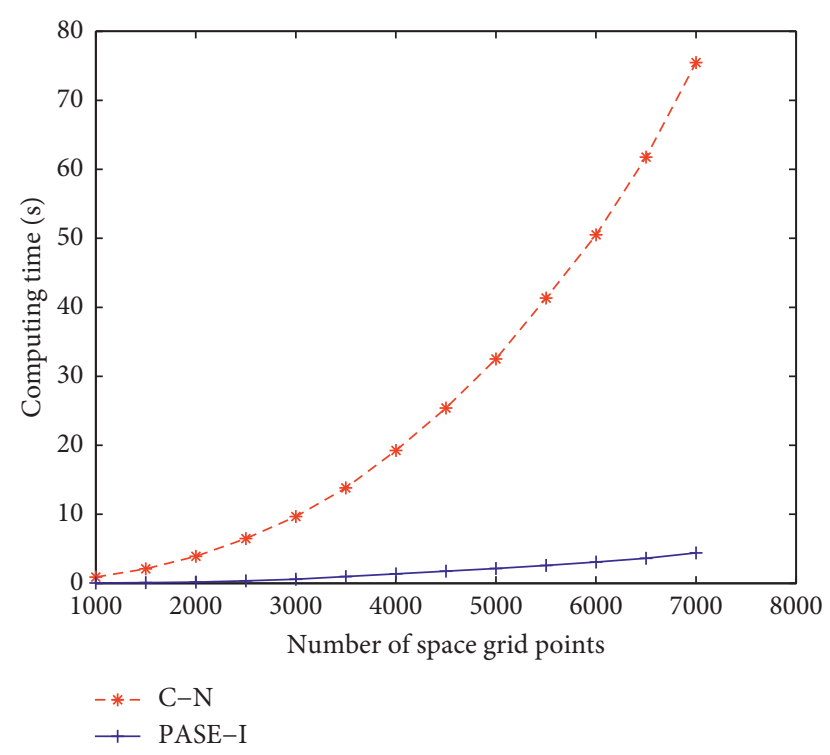

FIGURE 17: Comparison on the computing time of the C-N scheme and the PASE-I scheme.

\section{Conclusion}

The PASE-I and PASI-E difference methods with intrinsic parallelism for the Burgers-Fisher equation are linearly absolute stable. The solutions of schemes are existing and unique, with 2-order spatial accuracy and 2-order time accuracy. Numerical experiments verify the theoretical analysis. Numerical experiments show that PASE-I and PASI-E difference methods have ideal computational accuracy and obvious parallel computational properties. Their computational efficiency are much higher than that of the classical implicit difference method. PASE-I and PASI-E parallel difference methods for the Burgers-Fisher equation are efficient for various types of parallel computing systems. Especially when the space fraction is large enough, the methods have obvious localization characteristics in computation and communication. They are very suitable for application on massive parallel computing systems with distributed storage. PASE-I and PASI-E difference methods with intrinsic parallelism in this paper can also be extended to higher-dimensional problems, solving the high-dimensional Burgers-Fisher equation.

\section{Data Availability}

The data used to support the findings of this study are included within the article.

\section{Conflicts of Interest}

No potential conflicts of interest were reported by the authors.

\section{Acknowledgments}

This work was supported by the Subproject of Major Science and Technology Program of China (Grant no.
2017ZX07101001-01) and the Fundamental Research Funds for the Central Universities (Grant no. 2018MS168).

\section{References}

[1] R. E. Mickens and A. B. Gumel, "Construction and analysis of a non-standard finite difference scheme for the Burgers-Fisher equation," Journal of Sound and Vibration, vol. 257, no. 4, pp. 791-797, 2002.

[2] D. Kaya and S. M. El-Sayed, "A numerical simulation and explicit solutions of the generalized Burgers-Fisher equation," Applied Mathematics and Computation, vol. 152, no. 2, pp. 403-413, 2004.

[3] H. N. A. Ismail and A. A. A. Rabboh, "A restrictive padé approximation for the solution of the generalized Fisher and Burger-Fisher equations," Applied Mathematics and Computation, vol. 154, no. 1, pp. 203-210, 2004.

[4] M. Moghimi and F. S. A. Hejazi, "Variational iteration method for solving generalized Burger-Fisher and Burger equations," Chaos, Solitons \& Fractals, vol. 33, no. 5, pp. 1756-1761, 2007.

[5] C. H. Zhang and Z. Zhang, "Application of the enhanced modified simple equation method for Burger-Fisher and modified Volterra equations," Advances in Difference Equations, vol. 2017, no. 1, p. 145, 2017.

[6] J. C. Cheng, Mathematical Physics Equations and Theirs Approximate Methods, Science Press, Beijing, China, 2017, in Chinese, 2nd edition.

[7] H. N. A. Ismail, K. Raslan, and A. A. Abd Rabboh, "Adomian decomposition method for Burger's-Huxley and Burger'sFisher equations," Applied Mathematics and Computation, vol. 159, no. 1, pp. 291-301, 2004.

[8] E. Babolian and J. Saeidian, "Analytic approximate solutions to Burgers, Fisher, Huxley equations and two combined forms of these equations," Communications in Nonlinear Science and Numerical Simulation, vol. 14, no. 5, pp. 1984-1992, 2009.

[9] V. Chandraker, A. Awasthi, and S. Jayaraj, "Numerical treatment of Burger-Fisher equation," Procedia Technology, vol. 25, pp. 1217-1225, 2016.

[10] O. P. Yadav and R. Jiwari, "Finite element analysis and approximation of Burgers'-Fisher equation," Numerical Methods for Partial Differential Equations, vol. 33, no. 5, pp. 1652-1677, 2017.

[11] D. J. Evans and A. R. B. Abdullah, "Group explicit methods for parabolic equations," International Journal of Computer Mathematics, vol. 14, no. 1, pp. 73-105, 1983.

[12] B. L. Zhang and W. Z. Li, "On alternating segment CrankNicolson scheme," Parallel Computing, vol. 20, no. 6, pp. 897-902, 1994.

[13] B. L. Zhang, T. X. Gu, and Z. Y. Mo, Principles and Methods of Numerical Parallel Computation, National Defense Industry Press, Beijing, China, 1999, in Chinese.

[14] Z. Han, H. Y. Fu, and L. J. Shen, "Pure alternating segment explicit-implicit method for the diffusion equations," International Journal of Computer Mathematics, vol. 51, no. 1-2, pp. 55-68, 1994.

[15] Y. L. Zhou, "Difference schemes with intrinsic parallelism for quasi-linear parabolic systems," Science in China Series A: Mathematics, vol. 40, no. 3, pp. 270-278, 1997.

[16] P. Bjorstad and M. Luskin, Parallel Solution of Partial Differential Equations, Springer-Verlag, New York, NY, USA, 2000.

[17] A. Borhanifar and R. Abazari, "An unconditionally stable parallel difference scheme for telegraph equation," 
Mathematical Problems in Engineering, vol. 2009, Article ID 969610, 17 pages, 2009.

[18] Z. Q. Sheng, G. W. Yuan, and X. D. Hang, "Unconditional stability of parallel difference schemes with second order accuracy for parabolic equation," Applied Mathematics and Computation, vol. 184, no. 2, pp. 1015-1031, 2007.

[19] F. L. Qu and W. Q. Wang, "Alternating segment explicitimplicit scheme for nonlinear third-order KdV equation," Applied Mathematics and Mechanics, vol. 28, no. 7, pp. 973-980, 2007.

[20] G. W. Yuan, Z. Q. Sheng, and X. D. Hang, "The unconditional stability of parallel difference schemes with second order convergence for nonlinear parabolic system," Journal of Partial Differential Equations, vol. 20, no. 1, pp. 45-64, 2007.

[21] G. Y. Guo, S. J. Lü, and B. Liu, "Unconditional stability of alternating difference schemes with variable time steplengthes for dispersive equation," Applied Mathematics and Computation, vol. 262, pp. 249-259, 2015.

[22] M. Namjoo, M. Zeinadini, and S. Zibaei, "Nonstandard finitedifference scheme to approximate the generalized BurgersFisher equation," Mathematical Methods in the Applied Sciences, vol. 41, no. 17, pp. 8212-8228, 2018.

[23] R. F. Yan, X. Z. Yang, and S. Z. Sun, "Parallel computing method of pure alternative segment explicit-implicit difference scheme for nonlinear Leland equation," Annals of Applied Mathematics, vol. 34, no. 3, pp. 302-318, 2018.

[24] L. D. Herve and L. Brigitte, Partial Differential Equations: Modeling, Analysis and Numerical Approximation, Springer International Publishing, Cham, Switzerland, 2016.

[25] R. Jiwari, R. C. Mittal, and K. K. Sharma, "A numerical scheme based on weighted average differential quadrature method for the numerical solution of Burgers' equation," Applied Mathematics and Computation, vol. 219, no. 12, pp. 66806691, 2013.

[26] R. C. Mittal, R. Jiwari, and K. K. Sharma, “A numerical scheme based on differential quadrature method to solve time dependent Burgers' equation," Engineering Computations, vol. 30, no. 1, pp. 117-131, 2013.

[27] R. Jiwari, "A hybrid numerical scheme for the numerical solution of the Burgers' equation," Computer Physics Communications, vol. 188, pp. 59-67, 2015.

[28] A. Golbabai and M. Javidi, "A spectral domain decomposition approach for the generalized Burger's-Fisher equation," Chaos, Solitons \& Fractals, vol. 39, no. 1, pp. 385-392, 2009.

[29] Z. H. Xu and D. Q. Xian, "Application of exp-function method to generalized Burgers-Fisher equation," Acta Mathematicae Applicatae Sinica, English Series, vol. 26, no. 4, pp. 669-676, 2010.

[30] S. Kutluay and A. Esen, "A finite difference solution of the regularized long-wave equation," Mathematical Problems in Engineering, vol. 2006, Article ID 85743, 14 pages, 2006.

[31] G. H. Gao and Z. Z. Sun, "Two difference schemes for solving the one-dimensional time distributed-order fractional wave equations," Numerical Algorithms, vol. 74, no. 3, pp. 675-697, 2017.

[32] L. F. Wu and X. Z. Yang, "An efficient alternating segment parallel difference method for the time fractional telegraph equation," Advances in Mathematical Physics, vol. 2020, Article ID 6897815, 11 pages, 2020.

[33] P. Pacheco, An Introduction to Parallel Programming, Morgan Kaufmann, Burlington, MA, USA, 2011. 